(C) 1997 Cambridge University Press

\title{
The proper orthogonal decomposition of pressure fluctuations surrounding a turbulent jet
}

\author{
By R. E. A. ARNDT ${ }^{1}$, D. F. LONG ${ }^{2}$ AND M. N. GLAUSER \\ ${ }^{1}$ St. Anthony Falls Hydraulic Laboratory, University of Minnesota, \\ Minneapolis, MN 55414, USA \\ ${ }^{2}$ Fluidyne Engineering Corporation, Golden Valley, MN 55427, USA \\ ${ }^{3}$ Clarkson University, Potsdam, NY 13699-5725, USA
}

(Received 2 March 1996 and in revised form 17 December 1996)

It is shown that the pressure signal measured at the outer edge of a jet mixing layer is entirely hydrodynamic in nature and provides a good measure of the large-scale structure of the turbulent flow. Measurement of the pressure signal provides a unique opportunity to utilize proper orthogonal decomposition (POD) to deduce the streamwise structure. Since pressure is a scalar, a significant reduction in the numerical and experimental complexity inherent in the analysis of velocity vector fields results.

The POD streamwise eigenfunctions show that the structure associated with any frequency-azimuthal mode number combination displays the general characteristics of amplification-saturation-decay of an instability wave, all within about three wavelengths. High-frequency components saturate early in $x$ and low-frequency components saturate further downstream, indicative of the inhomogeneous character of the flow in the streamwise direction. Application of the POD technique allows the phase velocity to be determined taking into account the inhomogeneity of the flow in the streamwise direction. The phase velocity of each instability wave (POD eigenvector) is constant and equal to $0.58 U_{j}$, indicating that the jet structure is non-dispersive.

Using the shot-noise decomposition, a characteristic event is constructed. This event is found to contain evidence of both pairings and triplings of vortex structures. The tripling results in a rapid increase in the first asymmetric $(m=1)$ component. On average, pairing occurs once every four $U_{j} / D$ while tripling occurs once every $13 U_{j} / D$.

\section{Introduction}

This paper deals with the identification of large-scale structures in turbulent jets through an examination of the near-field pressure signal which is an outgrowth of studies going back more than 20 years (Arndt \& George 1974). The measurement procedure is based on the proper orthogonal decomposition (POD) originally proposed by Lumley (1967) for the study of spatial structure associated with inhomogeneous flows.

The measurements reported herein are based on work initiated in 1980 to study the influence of coherent structures on jet noise radiation using these earlier concepts. At the time no reliable method for deducing coherent structures in high-Reynolds-number flows in an unbiased manner was available. A review of the literature indicated that coherent structures were readily identified in clean jets at low Mach number and Reynolds numbers less than about $10^{5}$. However, subsonic jet noise measurements were made at higher Mach numbers and correspondingly higher Reynolds numbers as reviewed by Long \& Arndt (1984). Clearly, there was a need to deduce the presence of 
coherent structures at Reynolds numbers that are typical for jet noise experiments. The work of Professor Hussain and his co-workers supports this contention (Hussain 1983, 1986).

Three institutions were involved in a collaborative study of this issue. The approach was to develop a POD scheme using low-Reynolds-number experiments where comparisons with high-quality flow visualizations could be carried out. After the POD technique was developed, it could be applied to higher-Reynolds-number flows. A group at the Illinois Institute of Technology under the direction of Professor $\mathrm{H}$. Nagib carried out a series of careful flow visualizations using the smoke-wire technique developed at their laboratory (Drubka 1981). Professor W. K. George and his group at SUNY Buffalo developed the POD system using a multi-probe hot-wire technique (Glauser, Leib \& George 1985, 1987; Glauser \& George 1987a, b, 1992; Grinstein, Glauser \& George 1995). Professor R. E. A. Arndt and his group at the St. Anthony Falls Hydraulic Laboratory (SAFHL) carried out a series of jet noise experiments over a range of Reynolds number that spanned the values used in flow visualization and typical jet noise experiments (Long \& Arndt 1984). In this portion of the study, Reynolds number could be varied independently of Mach number by using a series of different nozzles ranging in size from $1.4 \mathrm{~mm}$ to $25 \mathrm{~mm}$. Every effort was made to ensure commonality of nozzle design and initial conditions in the three different laboratories. As the study progressed, it became clear that POD measurements at low Reynolds number but at high Mach number would be extremely difficult if not impossible because of the very small nozzle sizes involved. Thus it was decided to extend the POD technique to the pressure signal at the outer edge of the mixing layer in the jets used in the SAFHL studies. As a first step, we were able to carry out POD pressure measurements at moderate Reynolds number and low Mach number with and without jet excitation. The results of this effort were first published in a dissertation by Long (1985) and a conference proceedings article by Long \& Arndt (1985). Publication was delayed because of concerns about the proper selection of phase angle as outlined in $\S 6$ of this paper.

The implementation of POD to the pressure field offers significant advantages in several applications. For example, a natural extension of this work would be the application of POD to both hot and cold high-speed jets. Such experiments are presently underway at NASA-Langley where both the pressure and velocity fields are being examined via POD. The examination of the pressure field is of particular interest because of the relative simplicity in its measurement, which will provide practical opportunities for the application of POD-based control strategies (Carlson, Berkooz \& Lumley 1995).

Since the turbulence characteristics of a round jet are well documented, initial application of the POD to its pressure field provided the opportunity for comparisons with the results of various diagnostics (including POD) of the velocity field. Since the pioneering work of Crow \& Champagne (1971) it has been well established that largescale structures govern the mixing and entrainment of the entire jet, and may also play a significant role in the noise radiation process (Arndt \& George 1974; Michalke 1977; Moore 1977; Hussain 1983, 1986; Crighton 1981; Ho \& Huerre 1984; Liu 1989; Seiner \& Gilinsky 1995; Tam 1995). In addition, Ran \& Katz (1994) suggest that cavitation in turbulent jets is due to high-amplitude negative peaks in pressure that result from the pairing process.

There are numerous examples of application of POD to the velocity field from which comparisons with POD of the pressure field can be made. Moin (1984) and Moin \& Moser (1989) utilized channel flow simulations to provide the two-point correlation 
tensor. They found that the dominant eddy contributes as much as $76 \%$ to the turbulent kinetic energy. Glezer, Kadioglu \& Pearlstein (1989) applied an extended version of POD to a time-periodically forced mixing layer. Delville, Bellin \& Bonnet (1991) applied POD to a plane fully turbulent mixing layer. They found that $70 \%$ of the mean-square streamwise velocity was contained in the first three modes. Ukeiley et al. (1992) examined the multifractal character of the POD reconstructions of the instantaneous streamwise velocity fields in a lobed mixer flow. They found that the

higher POD mode contributions to the fluctuating velocity field were more multifractal in character, indicative of smaller scales.

Much of the recent work has been driven by interest in chaos and its relation to turbulence. Aubry et al. (1988) utilized the eigenfunctions of Herzog (1986) as a good set of basis functions in a dynamical systems approach to the near-wall region. Their results are not inconsistent with events such as bursting seen in experimental work. Zheng \& Glauser (1991) have used their jet eigenfunctions to develop a similar type of dynamical systems model for the jet mixing layer. They find clear evidence of pairs of vortices interacting in the streamwise direction resulting in a transfer of azimuthal to streamwise vorticity. Rajaee, Karlson \& Sirovich (1994) developed a low-dimensional model for a non-turbulent forced mixing layer. They found good agreement between their model coefficients and those obtained directly from experiment. A joint effort between CEAT/LEA Poitiers and Clarkson University has involved an examination of a fully turbulent mixing layer via a POD-based dynamical systems model (Ukeiley \& Glauser 1995; Delville 1995; Manceau 1995). The reconstructed velocity fields from their model capture the main streamwise and spanwise vortical structures known to exist in the mixing layer. Chambers et al. (1988) and Sirovich \& Rodriguez (1987) have applied this approach to Burgers equation and the Ginsburg-Landau equation respectively. Although these two applications are not in turbulent flows, both of the equations exhibit chaotic dynamics. For a more comprehensive review of the POD and its application in turbulent flows see Berkooz, Holmes \& Lumley (1993).

\section{Experimental procedure}

The experiments were carried out in a mini-anechoic chamber at the St. Anthony Falls Laboratory at the University of Minnesota. Its features are described by Long \& Arndt (1984). The chamber measures $2.2 \mathrm{~m}$ on a side and is lined with acoustic foam wedges that are $7 \mathrm{~cm}$ in length and are backed with fibreglass insulation. This provides anechoic conditions at frequencies above $1 \mathrm{kHz}$. The chamber has a porous front wall constructed with offset sections of acoustic foam so that there is no direct line of sight into the test chamber. The entrained flow is captured at the end of the chamber and recirculated through a carefully designed acoustically treated return duct and blower arrangement. The air for the jet is supplied from an air compressor. It passes through two filters, a pressure regulator, two mufflers and a $12.7 \mathrm{~cm}$ plenum just upstream of the contraction. Considerable development of the mufflers was needed to ensure that the flow was free of acoustic disturbances in the frequency range of interest.

The measurement of the cross-spectral density matrix was conducted using a $2.54 \mathrm{~cm}$ nozzle operated an exit speed of $23 \mathrm{~m} \mathrm{~s}^{-1}$. These measurements were complemented with other near-field measurements using a $7.1 \mathrm{~mm}$ nozzle. Both nozzles have a wall contour specified by a fifth-order polynomial. The larger nozzle contraction ratio is 25 and the length-to-diameter ratio is 1.25 . The smaller nozzle attaches directly to the end of the larger contraction and has a length-to-inlet diameter ratio of 1.0 and a 
contraction ratio of 12.8 . The overall contraction ratio of the two nozzles in tandem is 321 . The exit turbulence intensity for both nozzles was below $0.15 \%$. The boundary layer at the exit of the $2.54 \mathrm{~cm}$ nozzle is laminar with a momentum thickness of $0.1 \mathrm{~mm}$. This was determined using a procedure described by Long, Kim \& Arndt (1985).

Two B and $\mathrm{K} 3 \mathrm{~mm}$ microphones are used to measure the pressure fluctuations. The data were sampled simultaneously at $5.12 \mathrm{kHz}$ (resulting in a bandwidth of $20 \mathrm{~Hz}$ ) by a two-channel, 12-bit Preston A/D converter and were processed with a DEC PDP 11/34 mini-processor. The cross-spectral density was determined by a software FFT using 100 ensembles each containing 256 points. These spectra were stored on magnetic disk for future processing. A discussion of the use of microphones to measure hydrodynamic pressure fluctuations can be found in Long (1985). A detailed discussion of the measurement grid and sampling rate is given in $\S 5.2$, which is preceded by a detailed discussion of the application of POD to the pressure field.

\section{Preliminary velocity measurements}

In order to facilitate comparisons between the POD pressure application presented here and other jet studies where POD was applied to the velocity field, some velocity field measurements were necessary. Hence the spectra of the streamwise velocity fluctuations on the centreline within the potential core and within the mixing region were measured in order to describe the global nature of the jet flow.

Most velocity measurements were made with a TSI T1.5 subminiature hot-wire probe. Because of the very small spatial resolution that was necessary to measure the instability frequency of the exiting laminar shear layer, even smaller probes were manufactured in house. This was motivated by the concerns raised by Hussain \& Zaman (1978) that the probe body of the probe could produce a strong shear tone when introduced into the shear layer close to the tip. Only the tips of the wire supports in the smaller probe are inserted into the shear flow allowing a measurement of the instability frequency that is not influenced by the probe itself. The TSI probe uses a $2 \mathrm{~mm}$ long segment of $4 \mu \mathrm{m}$ diameter tungsten wire with a cold resistance of $6 \Omega$, whereas the smaller probes have a $1 \mathrm{~mm}$ segment of $4 \mu \mathrm{m}$ wire having a cold resistance of $3 \Omega$. A TSI 1050 series anemometer was used with either probe and the \# 1 bridge.

It was concluded from these preliminary velocity measurements that the high wavenumbers (small scales) show almost the same character and amplitude throughout the flow, independent of location, as evidenced by the collapse of the data in the inertial subrange. On the other hand, the low wavenumbers behave differently depending on the spatial location. This is evidenced by the increasing amplitude as the hot-wire probe was moved downstream. In other words, the large-scale structure is somewhat inhomogeneous in the downstream direction. Since Fourier methods are only optimal for homogeneous directions a more general approach is desirable, hence the implementation of the proper orthogonal decomposition.

\section{Spectral behaviour of pressure fluctuations}

\subsection{A simple model}

It is known from numerous flow visualization experiments that the flow field can be roughly divided into three parts: the potential core, the shear layer, and the entrainment region. See Long et al. (1985) for a more complete description. Their photographs suggest that the entrainment region is basically an unsteady irrotational flow. The average boundary between the entrainment region and the shear region 
occurs at roughly an angle of $10^{\circ}$. Since the POD is going to be applied in the irrotational entrainment region, it is necessary to first study the spectral behaviour of the pressure fluctuations in this region. These measurements are complementary to the measurements of pressure fluctuations within a turbulent shear flow as studied previously by George, Beuther \& Arndt (1984) and by Jones et al. (1979).

Since an exact solution for the pressure field surrounding a jet is not available, a simple model that will display the essential features of spatial decay and spectral variations is desired. To do this we look to the point-source solution of the spherical wave equation that is consistent with a turbulent free shear flow. It is recognized that this solution will not model the magnitude of the pressure fluctuations resulting from a turbulent jet since only a single source is considered but it serves to satisfy the desired objectives stated above.

In irrotational flows, the relationship between pressure and velocity is described by the unsteady Bernoulli equation.

$$
\frac{P-P_{\infty}}{\rho}=\frac{\partial \phi}{\partial t}-\frac{\nabla \phi \cdot \nabla \phi}{2},
$$

where $P_{\infty}$ is the pressure far from the flow and $\phi$ is the velocity potential. The pressure fluctuations described by (4.1) can be divided into two parts: propagating or acoustic fluctuations that are in phase with the velocity fluctuations, and non-propagating or hydrodynamic fluctuations that are $90^{\circ}$ out of phase with the velocity. Acoustic disturbances occur far away from a source in the active or far field. Hydrodynamic disturbances occur near the source in the reactive or near field. The terms near field and far field are somewhat vague and need to be given a more formal definition.

In the usual acoustic approximation, the convective term in the Bernoulli equation is neglected. This approximation is valid in the far field and, as will be shown, is also valid in the near field if one does not get too close to the source. It is assumed that the turbulent shear flow is composed of a finite number of individual sources. The pertinent question to ask is what is the nature of the resulting pressure outside the source region?

In unbounded turbulence (shear flows for instance) there can be no sources of mass and no unbalanced forces so the solution of the spherical wave equation must have a quadrupole character (Lighthill 1954). The appropriate solution for the velocity potential is (Morse \& Ingard 1968)

$$
\phi=\frac{\partial^{2}}{\partial r^{2}}\left\{\frac{-\mathrm{i} q R_{o}^{2}}{4 \pi r} \mathrm{e}^{\mathrm{i}(\omega t-k r)}\right\},
$$

where $R_{o}$ is the source size and $q$ is the source strength. For simplicity, the boundary condition for an axial quadrupole,

$$
-\left.\frac{\partial \phi}{\partial r}\right|_{r=R_{o}}=\mathrm{i} U_{o} \cos ^{2} \theta \mathrm{e}^{\mathrm{i} \omega t}
$$

is used to eliminate $q$ in favour of $U_{o}$, which is the acoustic source velocity. After performing the differentiation the pressure is obtained by substitution into equation (4.1). The solution for the mean-square pressure (which is the necessary quantity for comparison with experimental measurements) is found as

$$
I=\frac{\left(P-P_{\infty}\right)^{2}}{\rho_{o} a_{o}}=\rho_{o} a_{o} U_{o}^{2}\left(k R_{o}\right)^{2}\left[\frac{R_{o}}{r}\right]^{6}\left|\frac{2+2 \mathrm{i} k r+(\mathrm{i} k r)^{2}}{B}\right|^{2},
$$


where

$$
B=6-3\left(k R_{o}\right)^{2}+\mathrm{i}\left[6 k R_{o}-\left(k R_{o}\right)^{3}\right]
$$

and $\rho a_{o}$ is the acoustic impedance.

The assumption is now made that the product $\left(k R_{o}\right)$ is a constant. This follows from assuming that long-wavelength disturbances are associated with large sources and short-wavelength disturbances are associated with small sources. The product $\left(k R_{o}\right)$ is probably not exactly constant, but for the present discussion this approximation appears valid. The value of this constant is unknown. Since variations in the intensity rather than the absolute magnitude are sought, its precise value is not needed.

In the limit as the product of wavenumber and distance becomes large $(k r \gg 1)$ the mean-square pressure shows far-field behaviour. This implies that

$$
I \propto \rho_{o} a_{o} U_{o}^{2}(k r)^{-2} .
$$

It should be noted that this is the necessary form for far-field fluctuations in a threedimensional field. The intensity must decay as $r^{-2}$ in space in order to conserve the propagation of energy. Since (4.6) represents the radiation field, wavenumber can be transformed into frequency by

$$
\omega=a_{o} k,
$$

resulting in the intensity having a spectral decay of $\omega^{-2}$. This is in qualitative agreement with measured noise spectra.

In the limit as the project of wavenumber and distance becomes small $(k r \ll 1$, but keeping $r>R_{o}$ ) the intensity shows near-field behaviour. Performing this limit operation on (4.5) shows that

$$
I \propto \rho_{o} a_{o} U_{o}^{2}(k r)^{-6} .
$$

The source velocity, $U_{o}$, can be approximated as a typical turbulence intensity in the jet shear layer. If broken down into its spectral components this implies that

$$
U_{o}^{2} \sim k E(k),
$$

where $E(k)$ is the turbulent energy spectrum. Inspection of preliminary velocity data indicated that the spectrum can be divided into an energy-containing region where the spectral level is relatively flat and an inertial subrange which shows a $k^{-5 / 3}$ dependence.

The variation of the pressure intensity follows from inserting this turbulent energy spectrum into (4.9). It follows directly that in the energy-containing region at constant wavenumber, the intensity will have a spatial decay of

$$
I \propto r^{6} \quad(k=\text { const }),
$$

while in the inertial subrange the intensity will have a spectral variation (at constant $r$ ) of

$$
I \propto k^{-2 / 3} k^{-6} \propto k^{-6.67} \quad(r=\text { const }) .
$$

\subsection{Experimental verification}

To test these ideas, a microphone was traversed radially outward from the edge of a jet as shown in figure 1. The power spectrum of the measured pressure, $S(\omega)$, was obtained at various locations with frequencies transformed into acoustic wavenumbers by equation (4.7). This allows one to look at the spatial decay of the pressure fluctuations at a constant wavenumber or at the wavenumber spectrum at a constant radial distance, $y$. The variable $y$ is taken as the distance from the microphone face to 


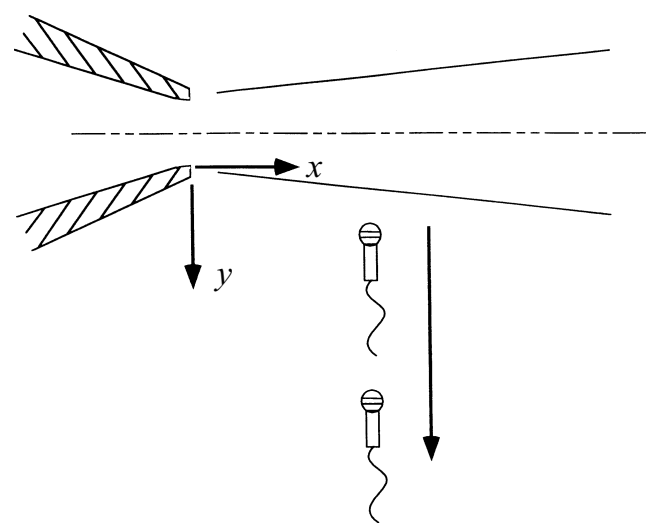

FIGURE 1. The experimental set-up designed to test the near-field pressure model.

the centre of the mixing region. The centre of the mixing region was determined from hot-wire measurements and is approximately equal to the nozzle lip line. Other definitions for $y$ were tried such as from the centreline of the jet and from the edge of the shear layer but the centre of the mixing region seemed to produce the best results.

These results qualitatively agree with the simple model presented above. This is illustrated in figures $2(a)$ and $2(b)$ for a low-Mach-number case where far-field behaviour can be neglected. The data in these figures corresponded to a $2.54 \mathrm{~cm}$ jet operated at $U=23 \mathrm{~m} \mathrm{~s}^{-1}$. Two axial distances were chosen: $x=1.5 D$, and $x=2.25 D$. To explain how to interpret these figures the reader's attention is first drawn to figure $2(a)$. The solid curves represent the individual spectra measured by the microphone as it is moved radially outward. Wavenumbers are non-dimensionalized by the radial distance $y$ on the abscissa. As the microphone is moved further away from the shear region, the intensity of the fluctuations becomes less and the spectra become more broad band. The data points are the amplitudes of individual spectra at constant values of $k D$ or $S t_{D}$. In particular, the $S t_{D}=0.5$ data points represent the fluctuations at the peak of each individual spectrum. A best-fit line through these points exhibits a $(k y)^{-6}$ decay which is consistent with the expected $(y)^{-6}$ behaviour for constant $k$ in the energy-containing region. The $S t_{D}=1.0$ data points represent the inertial-subrange fluctuations and the slope of these data has the expected $k^{-6.67}$ behaviour for constant $y$. Moving to figure 2(b) for the axial distance of $x=2.25 D$ these slopes are seen more clearly. Note that the data points in figure $2(b)$ correspond to the same values of $S t_{D}$ as shown in figure $2(a)$. Since the measurement takes place further downstream, the energy-containing scales have moved to lower wavenumber $\left(S t_{D}=0.22\right.$ data points) and the inertial subrange is shown to the right of this.

Attention should now be focused on the low-wavenumber region of figure $2(b)$. The slope through any of these data points (for instance the open circles) is less than the expected value of $y^{-6}$. The reason for this is depicted pictorially on figure 3 . As shown, this is primarily an effect of the inhomogeneous nature of the shear flow. Generally it is expected that small sources will occur in the shear layer near the nozzle exit and larger sources will occur further downstream. In particular, large sources will produce low-wavenumber (long wavelength) disturbances. Figure 3 shows that as the microphone is moved from $y$ to $2 y$ the actual distance from the low-wavenumber source changes by a smaller amount. Thus, the decay rate is expected to be less than $y^{-6}$. It is obvious from the geometry of the situation that the spatial decay rate depends on how far downstream the source is located. For lower and lower wavenumbers the 

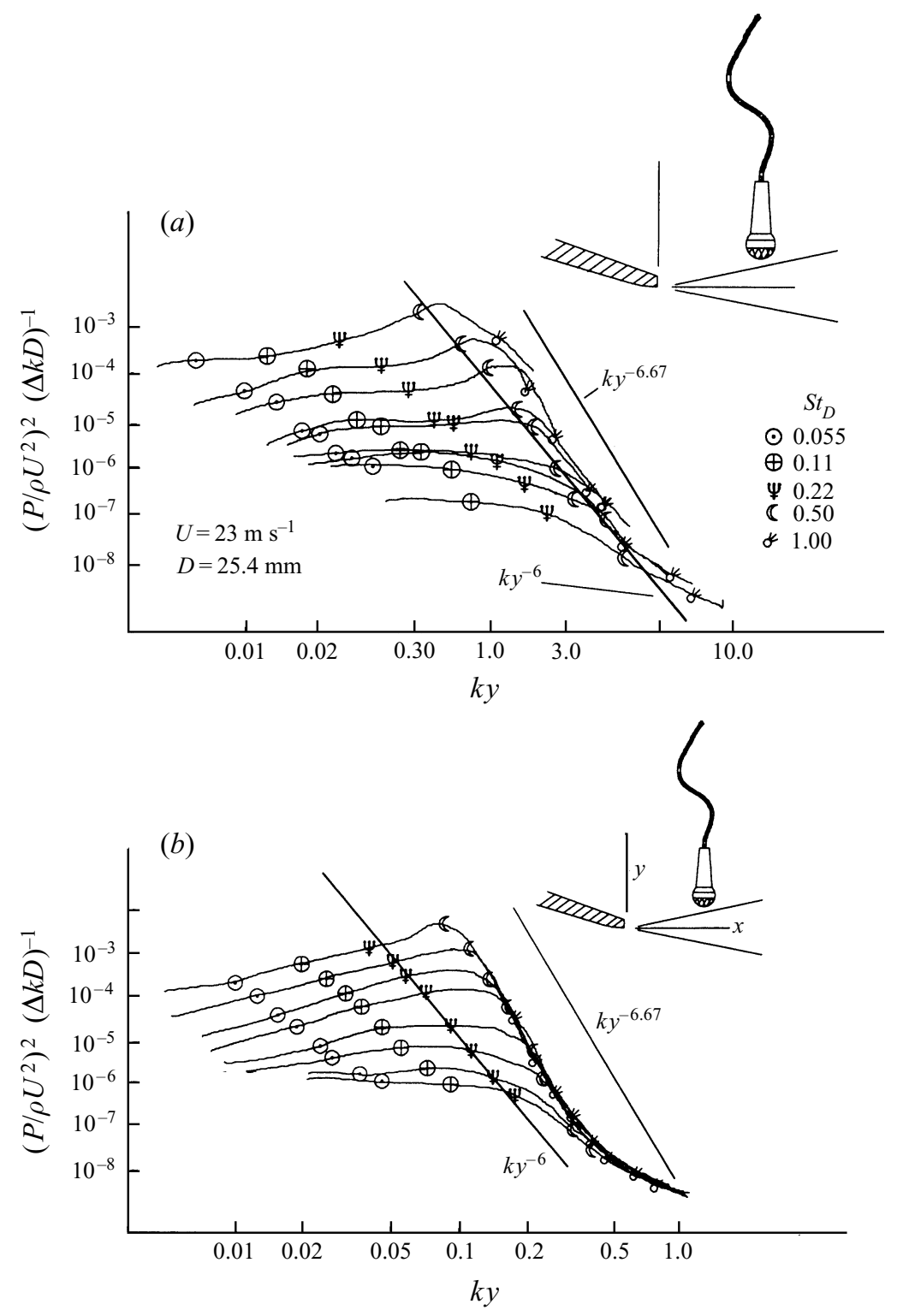

FiguRE 2. Evolution of near-field pressure fluctuations at low Mach number for $S t_{D}=0.055$ to $S t_{D}=1.00$ at $(a) x=1.5 D,(b) x=2.25 D$.

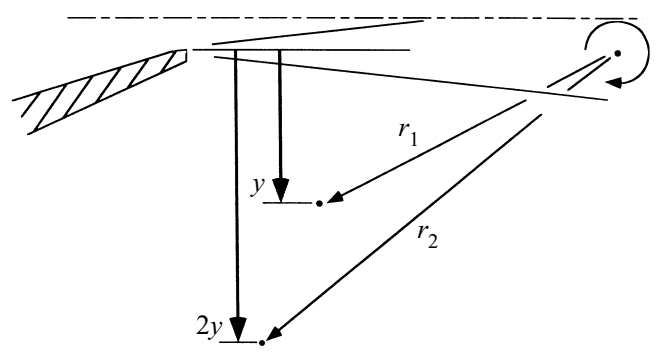

FIGURE 3. Relationship between changes in $y$ and changes in the actual source-observer distance. 


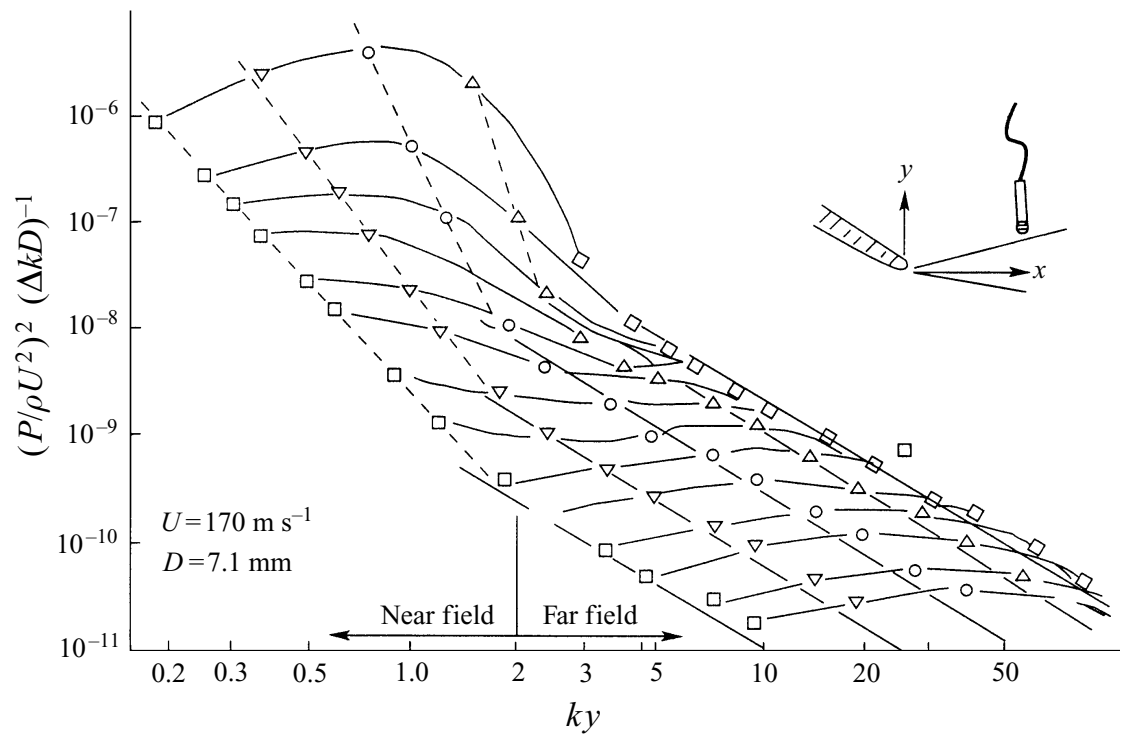

FIGURE 4. Evolution of near-field pressure fluctuations into far-field pressure fluctuations at moderate Mach number, $M=0.52$ and $x=3 D . \square, S t_{D}=0.04 ; \nabla, S t_{D}=0.08 ; \bigcirc, S t_{D}=0.17$; $\triangle, S t_{D}=0.33 ; \diamond, S t_{D}=0.67$.

spatial decay rate will become flatter and flatter in terms of the variable $y$. If the exact location of the source were known, the correct distance could be chosen to obey the $r^{-6}$ decay law. $\dagger$

Now consider a case where the Mach number is high enough so that far-field pressure fluctuations will occur with sufficient amplitude to be measurable. This is shown on figure 4 where the Mach number is $M=0.52$. The near-field characteristics are similar to the low-Mach-number case in the low-wavenumber region. Rather than the expected $k^{-6.67}$ decay at higher wavenumbers, the influence of the radiated acoustic field is seen, as a result of the higher Mach number. This is a consequence of the intensity of the radiated field scaling as $M^{5}$. The far field shows $y^{-2}$ behaviour at all frequencies (wavenumbers). It is interesting to note that there is a very sharp dividing line at $k y=2$ between near-field and far-field behaviour. When $k y>2$, the probe sees far-field fluctuations and when $k y<2$, the probe sees near-field fluctuations. The conclusion is that the dividing line between near field and far field is frequency dependent; it is not a rigidly fixed location in space. This also implies that far-field behaviour can be observed much closer to the jet, consistent with present thought. Most far-field noise experiments are conducted at a distance on the order of 100 diameters from the nozzle exit. However, depending on the frequency of interest the microphone may be placed much closer as long as the criterion $k y>2$ is maintained.

This observation may be useful where correlation techniques are used to pinpoint source locations. The accepted method is to place microphones on a polar arc centred around the nozzle exit. Details of this procedure can be found in Fisher, Harper-

$\dagger$ As pointed out by a reviewer, the reverse statement is also correct. With the assumption that the $r^{-6}$ law is valid, the exact downstream location of the source can be calculated. This could provide a unique way to determine the source.

* In principle $k y$ should be corrected for Doppler shift by a factor of $\left(1+M_{C} \cos \theta\right)^{-1}$ where $M_{C}$ is the convective Mach number (in this case $M_{C}$ is approximately 0.3 ) and $\theta$ is the angle between the observation point and the source. Unfortunately $\theta$ is an unknown function of $y$, see figure 3 . 


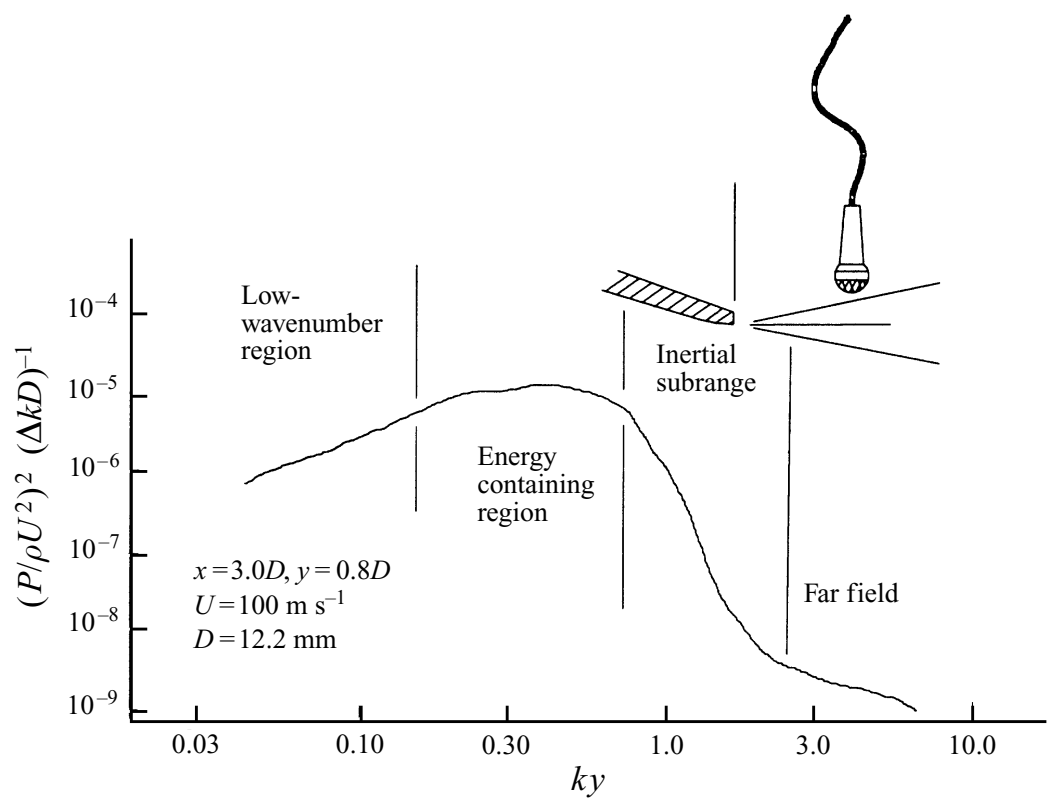

FIGURE 5. An example of the pressure spectrum showing all four regions.

Bourne \& Glegg (1977). It was used by Kim (1983) and Long et al. (1985) to determine the source locations. It was found that for frequencies below $S t_{D}=0.5$ the technique was not very accurate because the phase angle of the correlation signal decreases as frequency is decreased. In other words, at low frequency the difference in phase from the source to each of the microphones is not very large and cannot be measured accurately. By moving the microphones inward towards the limiting value of $k y=2$ it may be possible to achieve greater accuracy, especially at frequencies where the jet radiates the bulk of the noise.

By picking an intermediate value of Mach number all four spectral regions of the pressure fluctuations can be seen. This is shown on figure 5. The spectrum for values of $k y<0.2$ shows low-wavenumber behaviour where the spatial decay is somewhat less than $y^{-6}$. For $0.20<k y<0.8$ the pressure fluctuations show $y^{-6}$ behaviour characteristic of the energy-containing region being directly in front of the microphone. For $0.8<k y<2.0$, the pressure fluctuations are due to inertial-subrange turbulence, and for $k y>2.0$ the pressure fluctuations are acoustic.

These preliminary measurements clearly indicate that the most energetic part of the spectrum results from the turbulence that is directly in front of the microphone. This is an important point. It means that there is a direct link between the measured pressure and the location of turbulence in the shear layer. This shows that the scalar pressure field near the edge of the mixing layer is a significant dynamical quantity and is therefore an appropriate variable to decompose using the POD.

\section{Structure of near-field pressure fluctuations}

\subsection{Near-field pressure and large scales}

In order to relate the features of the large-scale structure of a jet to the hydrodynamic pressure field, we must consider the effect of 'wavenumber filtering' of the velocity fluctuations. In analysing the Green's function solution of the Poisson equation, 
George et al. (1984) found that the mean-square pressure is related to the Reynolds stress by a weighing function $W(k)$,

$$
W(k)=\frac{(4 \pi)^{2}}{|k|^{4}} .
$$

The nature of the rapid roll-off of this weighting function indicates that the pressure spectrum will be dominated by the larger turbulent scales. This is termed wavenumber filtering.

This means that the pressure signal is dominated by the larger scales and will be correlated over larger distances, implying that a coarser grid can be used to collect information on the pressure fluctuations than can be used for the velocity field. In fact, Fuchs (1972) showed that only the first few azimuthal modes $(m=0,1,2)$ were important in the pressure field within the mixing region. More recently Glauser \& George (1987b) and Grinstein et al. (1995) have shown that a greater number of azimuthal modes $(m=0-6)$ are important in the velocity field in the mixing region which is also consistent with (5.1). Although the analysis of George et al. (1984) was for the homogeneous case, it is expected that similar results will apply to the nonhomogeneous case as well. For this reason, it can be assumed that the pressure fluctuations in the axial direction will also be correlated over a longer distance. Hence sufficient information can be obtained by using an axial grid spacing roughly equivalent to that required to describe the low-order azimuthal modes.

\subsection{Application of the proper orthogonal decomposition to the near-field pressure signal}

As has been pointed out previously, the integral nature of the Poisson equation suggests that the pressure sampled at any axial position will be representative of the global structure of the events in the shear layer at that location. With this in mind, the pressure field is sampled just outside the jet at relatively few locations to obtain a 'picture' of the large structure without having to resort to sampling the velocity at a multitude of points within the shear region, cross-correlating and then performing the POD. This procedure does not yield 'characteristic eddies', but it does give more quantitative information on the size and orientation of the correlated regions.

We consider the jet to be stationary in time, periodic in the azimuthal variable, $\theta$, and inhomogeneous in the axial variable, $x$, (figure 6). We can write the random pressure signal as

$$
P=P(x, \theta, t) .
$$

It should be emphasized that the radial inhomogeneities have been integrated out because of the character of the pressure signal. The proper orthogonal decomposition theorem (also known as the Karhunen-Loeve expansion), interpreted in terms of the pressure signal states that $P(x)$ has an orthogonal decomposition (Loeve 1978)

$$
P(x)=\sum_{n=1}^{\infty} \lambda_{n} \zeta_{n} \psi_{n}(x),
$$

with $\quad \int_{x} \psi_{n}(x) \psi_{n^{\prime}}(x) \mathrm{d} x=\delta_{n n^{\prime}}, \quad E\left(\zeta_{n} \zeta_{n^{\prime}}\right)=\delta_{n n^{\prime}}$

if, and only if, the $\left|\lambda_{n}\right|^{2}$ are the proper values and the $\psi_{n}(x)$ are the proper functions of its covariance, where $E(\bullet)$ implies 'expected value'. The proper values and proper functions (hereafter called eigenvalues and eigenfunctions) represent the decomposition 

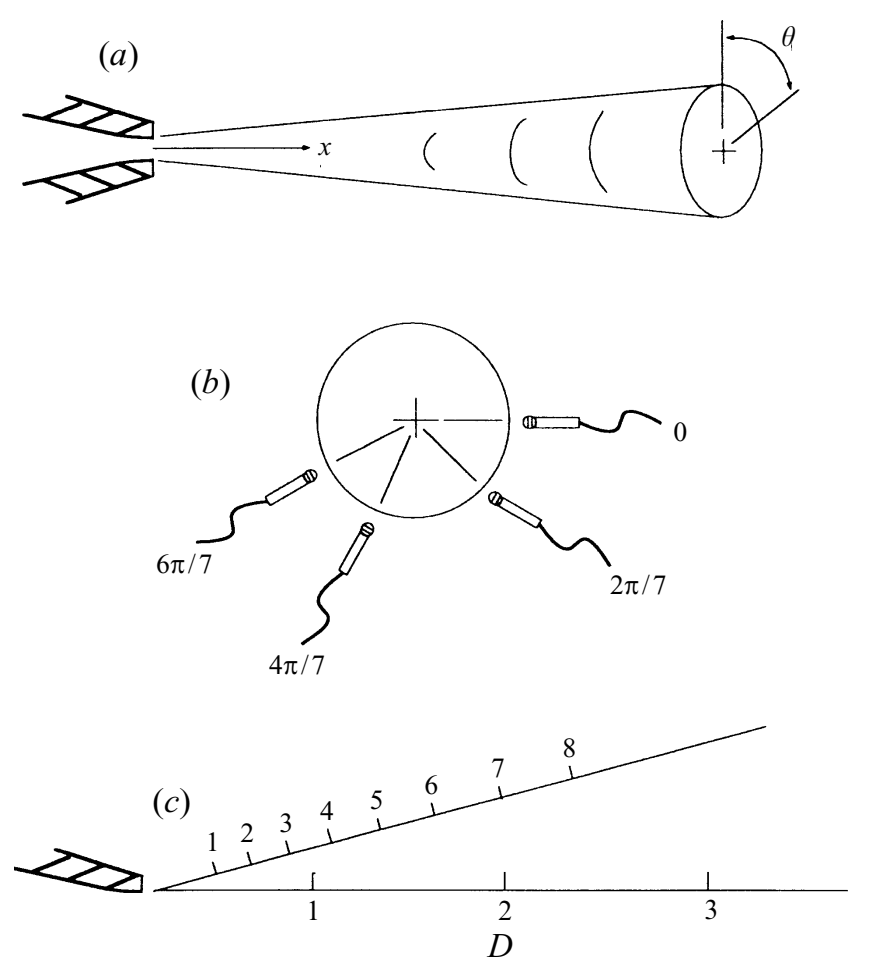

FiguRE 6. Cross-spectral density measurement locations for input to the orthogonal decomposition: (a) general layout, $(b)$ azimuthal spacing, $(c)$ axial spacing.

of the axial direction into its orthogonal components, and are found from the solution to the equation

$$
\int R\left(x, x^{\prime}\right) \psi_{n}\left(x^{\prime}\right) \mathrm{d} x^{\prime}=\left|\lambda_{n}\right|^{2} \psi_{n}(x),
$$

where $R\left(x, x^{\prime}\right)$ is the covariance of the pressure signal. The dependence on $t$ and $\theta$ (or $\omega$ and $m$ ) has been suppressed for simplicity. The eigenvalue $\left|\lambda_{n}\right|^{2}$ represents the energy content and the eigenfunction $\psi_{n}$ represents the characteristic signal form of that frequency-mode number combination.

Stationarity in time implies that a Fourier transform may be used to decompose the time signal into its orthogonal components. That is, a cross-spectral density of the pressure signal is defined as

$$
\phi\left(x, x^{\prime}, \theta ; \omega\right)=\int_{-\infty}^{\infty} R\left(x, x^{\prime}, \theta ; t\right) \mathrm{e}^{-\mathrm{i} \omega t} \mathrm{~d} t .
$$

Symmetry and periodicity in azimuthal angle imply that a Fourier cosine series can be used to represent the $\theta$-direction. The spectral density determined from (5.6) is decomposed as

$$
\phi\left(x, x^{\prime}, \theta ; \omega\right)=\sum_{m=0}^{\infty} \Phi\left(x, x^{\prime} ; \omega, m\right) \cos m \theta,
$$

where $m$ is the azimuthal mode number and the complex coefficients of the series are defined by

$$
\Phi\left(x, x^{\prime}, \omega, 0\right)=\frac{1}{\pi} \int_{0}^{\pi} \phi\left(x, x^{\prime}, \theta ; \omega\right) \mathrm{d} \theta
$$


and by $\quad \Phi\left(x, x^{\prime} ; \omega, m\right)=\frac{2}{\pi} \int_{0}^{\pi} \phi\left(x, x^{\prime}, \theta ; \omega\right) \cos m \theta \mathrm{d} \theta$.

Performing these operations on the pressure signal allows us to write (5.5) as

$$
\int \Phi\left(x, x^{\prime} ; \omega, m\right) \psi_{n}\left(x^{\prime} ; \omega, m\right) \mathrm{d} x^{\prime}=\left|\lambda_{n}(\omega, m)\right|^{2} \psi_{n}(x, \omega, m) .
$$

For each frequency-azimuthal mode number combination, this equation is solved to determine the axial dependence. The numerical details, which are similar to those discussed in Moin \& Moser (1989), can be found in Long (1985).

The decomposition is intended to take place on the surface of an imaginary cone whose axis is coincident with the jet axis (figure $6 a$ ). The surface of the cone passes through the nozzle lip. The proper cone angle is not obvious. Some preliminary data were taken along a cone angle of $15^{\circ}$. Subsequent to these preliminary measurements, flow visualization indicated that a $10^{\circ}$ cone angle is more appropriate (Long et al. 1985). This is consistent with the measurements of Ko \& Davies (1971) and Peterson (1978). This two-dimensional decomposition $(x, \theta)$ of the scalar pressure field is considerably easier to obtain than the three-dimensional decomposition of the velocity field. The diameter of the jet used was $25.4 \mathrm{~mm}$ and the exit velocity was $23 \mathrm{~m} \mathrm{~s}^{-1}$, corresponding to a Reynolds number of $3.9 \times 10^{4}$.

As will be discussed, the required input for the POD is the cross-spectral density matrix of pressure fluctuations between $x=0.5 D$ and $x=3.0 D$. This range includes at least $90 \%$ of the energy in the frequency range of interest. Eight axial probe locations and four azimuthal intervals are chosen to provide adequate information up to the $m=2$ mode. The size of the matrix for this scalar problem dictates the number of eigensolutions that can be computed. If the eigenvalues are widely separated in magnitude, there is a firm basis for believing that the turbulent motion associated with the matrix is coherent. If the amplitude does not fall off rapidly with mode number, the matrix does not correspond to a coherent motion. For an $n \times n$ matrix, $n$ eigenvalues could be computed (eight in this experiment). However, Lumley (1970) indicates that probably no more than three or four will be necessary and possibly only one (the largest) will be sufficient. This has been experimentally verified for jet flow by Glauser et al. (1987).

The four azimuthal intervals are spaced as shown in figure $6(b)$. With the assumption that the jet structure is periodic in azimuthal angle it is not necessary to make measurements on the upper half of the figure. This simplification reduces the measurements by a factor of two with the only drawback being that the plus and minus spinning modes cannot be distinguished from one another; they are lumped into the same value. This is justified by the assumption that a well-designed jet experiment should have no preferential direction of swirl.

The eight axial locations are spaced to take advantage of the observation that small scales occur near the jet exit and large scales occur further downstream. The spacing between the probes is logarithmic and has been designed to roughly satisfy the folding frequency criterion. That is, there should be at least two probe locations per wavelength. The locations are shown in figure $6(c)$.

Using eight axial locations and four azimuthal locations indicates that 256 singlesided cross-spectra are necessary; however utilizing the symmetry properties of the FFT allows the number to be reduced to 144 double-sided spectra. The range of frequency over which accurate data is necessary lies between $S t_{D}=0.1$ and $S t_{D}=4.0$. This includes the column mode frequency of $450 \mathrm{~Hz}\left(S t_{D}=0.5\right)$ and the instability 


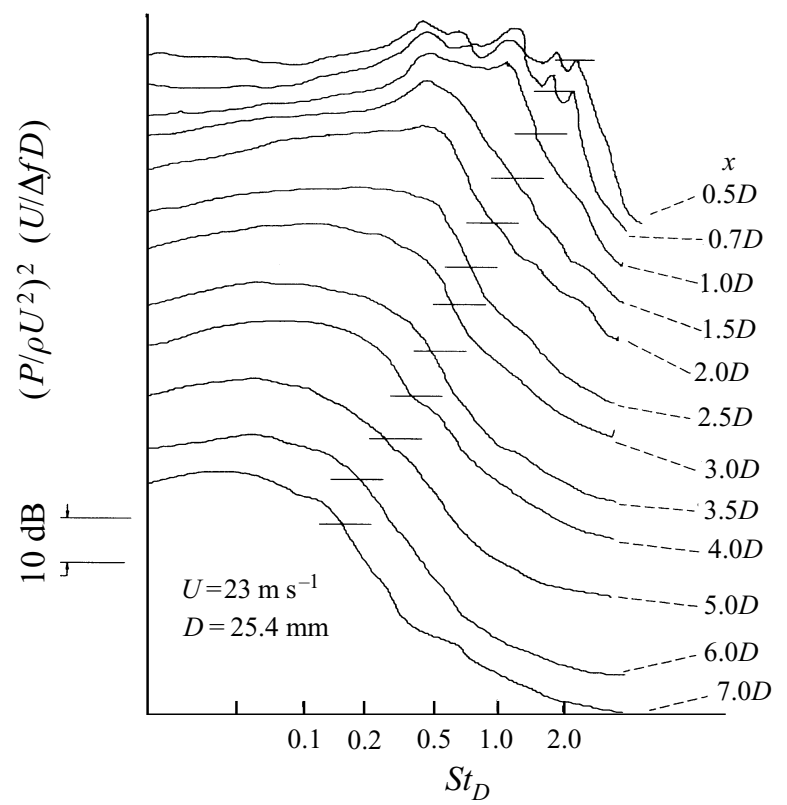

FiguRE 7. Axial variation of near-field pressure spectra along $\beta=15^{\circ}$ (the dark line intersecting each curve indicates a dimensionless level of $10^{-5}$ or $-50 \mathrm{~dB}$.

frequency of the shear layer of $3.6 \mathrm{kHz}\left(S t_{D}=4.0\right)$. Inspection of the near-field pressure spectra (figure 7) indicates that only pressure data below $S t_{D}=2.0$ are important, which corresponds to the first pairing. Based on these requirements, the sampling rate was chosen at $5.12 \mathrm{kHz}$. This produces a spectrum with a $20 \mathrm{~Hz}$ bandwidth, the most accurate range being one decade wide from $0.2<S t_{D}<2.0$.

The data collection was initiated after normal work hours to ensure constant conditions and minimum interference from extraneous noise sources from within the laboratory. Two sets of data were obtained; one for an unexcited jet and one for a jet excited at $3.0 \mathrm{kHz}$. Excitation at $3.0 \mathrm{kHz}$ results in a very stable signal with a spectrum containing discrete tones at $f_{e} / 2=1.5 \mathrm{kHz}$ and $f_{e} / 4=750 \mathrm{~Hz}$. Presumably these frequencies correspond to the pairings of axisymmetric ring vortices. This part of the experiment provides a good test for the POD.

\subsection{Discussion of unexcited jet results}

It has been suggested in the past that the axial direction can be rendered homogeneous if the data are scaled by $1 /\left(x-x_{0}\right)$ where $x_{0}$ is a virtual origin. The statistics can then be manipulated so that the axial direction appears homogeneous. That is, as the probe moves downstream, the spectral shape remains unchanged when normalized in this manner but gets shifted to lower frequencies. By applying the $1 /\left(x-x_{0}\right)$ scaling the spectra can be made to collapse onto one curve and the data appear homogeneous. If this assumption is valid, the required amount of data is reduced tremendously. However, preliminary velocity measurements indicated that this appears to be so only in the region downstream of $x=2.5 D$. The measured turbulence spectra for $x / D>3$ are apparently similar and could be scaled to collapse to a single curve. The preliminary near-field pressure spectra also show this trend. Figure 7 shows a sequence of spectra from $x=0.5 D$ to $x=7.0 D$ along a $15^{\circ}$ ray $\dagger^{\dagger}$ emanating from the nozzle lip. Clearly the

$\dagger$ Subsequent to obtaining these preliminary data the microphones were traversed along a $10^{\circ}$ ray as mentioned above in $\S 5.2$. 


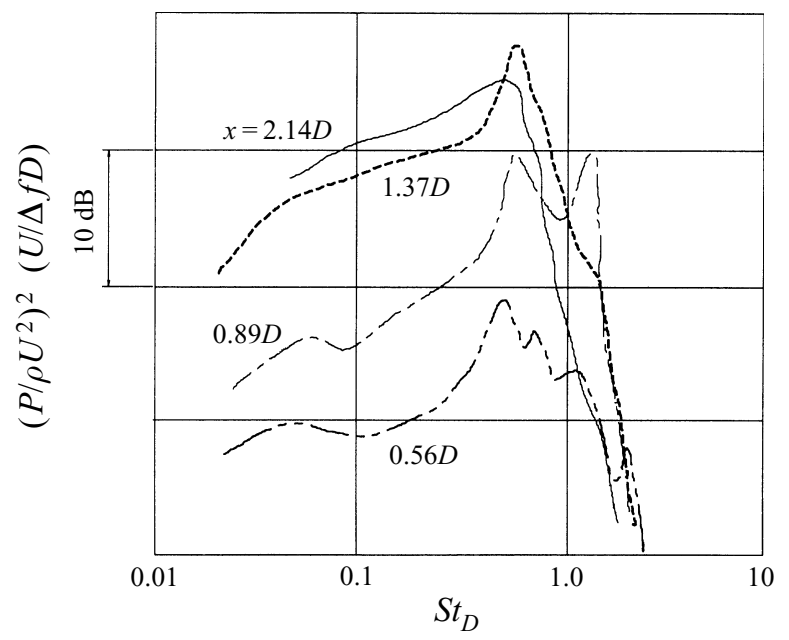

FIGURE 8. Examples of the near-field pressure spectra along $\beta=10^{\circ}$.

spectra have a similar shape only in the region downstream of $2.5 D$. Upstream of this position the spectra vary in shape. The conclusion is that the region between $x=0$ and $x=3 D$ must be considered inhomogeneous; scaling laws will not collapse the data onto one curve. Thus, the POD scheme has been restricted to this inhomogeneous range.

Of the 256 cross-spectra $(8 \times 8 \times 4)$, eight will be power spectra corresponding to $x=x^{\prime}$ and $\theta=0$. Since these data were obtained along a $10^{\circ}$ ray, which is different than figure 7, sample spectra are shown in figure 8. Note that the scaling has been changed. This sequence also shows clearly that scaling the data to make the turbulence appear homogeneous will not work in this region because the spectral shape changes. There are some curious peaks and discrete tones that should be mentioned briefly. In the spectrum corresponding to $x=0.56 D$, there are tones corresponding to the subharmonics of the natural instability frequency, $f_{i}=3.6 \mathrm{kHz}\left(f_{i} D / U=4.0\right)$. The $f_{i} / 2, f_{i} / 4$, and $f_{i} / 8$ tones, corresponding to the pairing process occurring further downstream, are evident. Moving downstream, some of these disappear and new ones emerge. In particular at $x=0.89 D$, there is, in addition to the $f_{i} / 8\left(f_{i} D / U=0.5\right)$ peak, an interesting tone at $S t_{D}=1.3$ which is not an even subharmonic of the instability frequency but an odd subharmonic equal to $f_{i} / 3$. This indicates that the merging process cannot be completely described by a pairing of vortices. Further downstream at $x=1.37 D$ only the $S t_{D}=0.5$ tone is dominant. Still further downstream the spectrum begins to approach the shape that can be scaled to appear independent of $x$. The sequence of events that produces these changes is embodied in the eigenfunctions of the POD obtained from application of (5.10). Before discussing this the eigenvalues must be considered because they are what determines whether or not there is sufficient coherent energy in a particular azimuthal mode to warrant looking at the details of the POD eigenfunctions. If a particular eigenvalue is large, the details of the corresponding eigenfunction are important. If that eigenvalue is small, the details are not important. If we sum up all these power spectra (for $x=x^{\prime}$ and $\theta=0$ ) according to the equation

$$
T(\omega)=\sum_{i=1}^{8} S_{i}(\omega) \mathrm{d} x_{i}
$$

we obtain the total energy spectrum. Note that is equivalent to summing the POD eigenvalues over all POD and azimuthal modes. This is shown in figure 9. It 


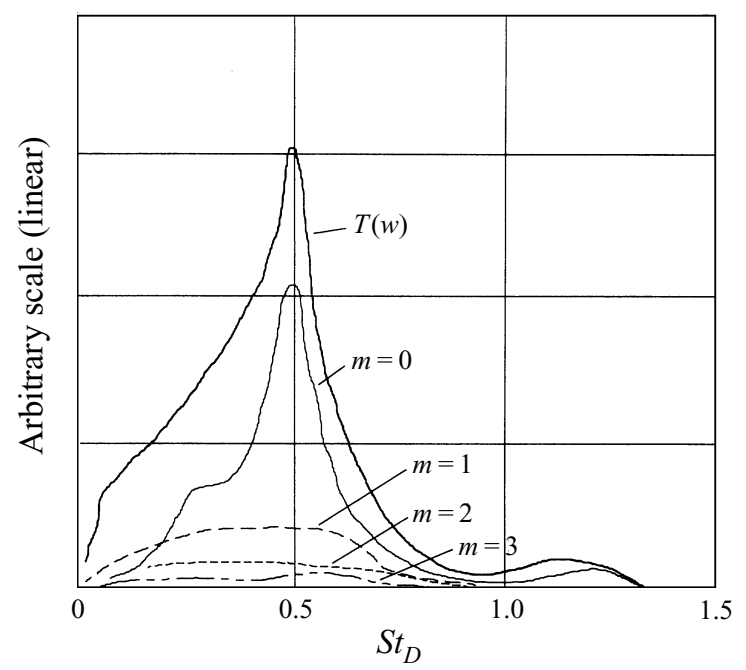

FIGURE 9. Total energy spectrum (equation (5.13)) and the dominant eigenvalue spectra for various azimuthal modes.

corresponds to the baseline situation. Most of the fluctuations in the total energy spectrum are found at the column mode frequency of $S t_{D}=0.5$. There is also a small hump at $S t_{D}=1.3$ which is a $1 / 3$ harmonic of the original instability wave which forms at the nozzle lip (cf. figure $10 d$ ).

The dominant POD eigenvalue spectrum of each azimuthal mode (i.e. $\left|\lambda_{1}(\omega ; m)\right|^{2}$, $m=0,1,2,3$ in (5.10)) is compared to the total energy spectrum in figure 9 to decide if this particular mode is significant or not. Clearly the $S t_{D}=0.5$ tone belongs to the axisymmetric class; the higher-order azimuthal modes contain significantly less energy at this frequency. If we sum up the coherent energy at this frequency represented by the first (largest) eigenvalue for each azimuthal mode, we find that this sum contains $98 \%$ at the total. If we integrate the $\left|\lambda_{1}\right|^{2}$ with respect to $\omega$, we find that $50 \%$ of the energy associated with the pressure fluctuations is contained in the $m=0$ mode; $23 \%$ is contained in the $m=1$ mode; $14 \%$ is contained in the $m=2$ mode; and $5 \%$ is contained in the $m=3$ mode. Thus, it is safe to say that all the energy associated with the pressure fluctuations is contained in the first four azimuthal modes of the largest POD eigenvalue spectra. In fact, the second POD eigenvalue spectrum of the axisymmetric mode is typically twenty times smaller than the first eigenvalue spectrum of the axisymmetric mode. This indicates that the pressure signal is filtering out the higher POD modes as well (as discussed earlier in this section). This is in stark contrast to the results of the decomposition of velocity fields (Moin \& Moser 1989; Glauser \& George 1987a, $b$; Grinstein et al. 1995).

This concept can also be seen in the bar charts shown on figure 10. Clearly, almost all the energy is located in the various modes of the first (largest) POD eigenvalue; the other eigenvalues contain no significant energy. It is interesting to note that the $m=0$ azimuthal mode is dominant at $S t_{D}=0.5$ and $S t_{D}=1.3$, indicating axisymmetric structures, while at other frequencies a significantly greater amount of energy is contained in the $m=1$ component relative to the $m=0$ component. This implies that the turbulence is less coherent at these frequencies indicating smaller turbulent structures.

The POD eigenvectors represent the spatial signal form associated with any particular frequency-azimuthal mode number combination. In view of the eigenvalue 

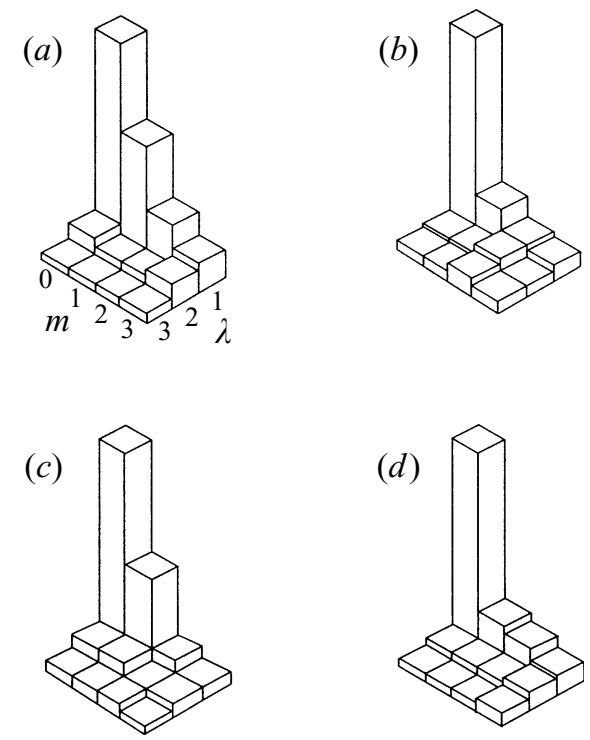

FIGURE 10. Eigenvalue histograms for various frequencies (each figure is normalized by the amplitude of the dominant eigenvalue at that frequency, $\left.\lambda_{1}\right)$ : (a) $S t_{D}=0.33 ;(b) S t_{D}=0.50$; (c) $S t_{D}=0.77 ;(d) S t_{D}=1.30$.

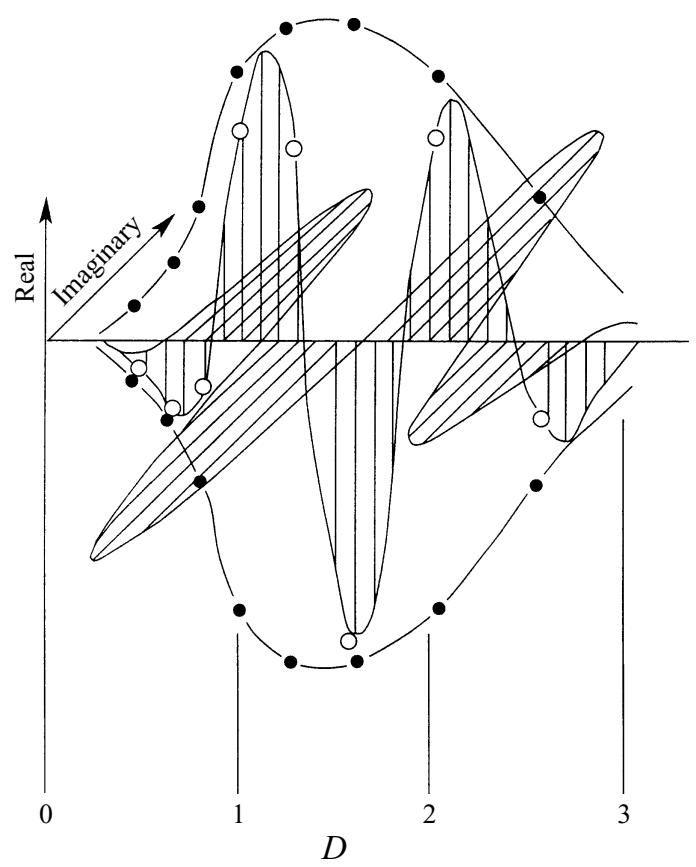

FIGURE 11. Eigenvector $\psi_{1}\left(S t_{D}=0.5, m=0\right)$, corresponding to the largest eigenvalue for the axisymmetric mode at the column mode frequency.

spectrum of figure 9, the most important frequency-azimuthal mode number combination is the one that corresponds to the column mode frequency of $S t_{D}=0.5$ and the axisymmetric or $m=0$ mode because this azimuthal mode contains most of the energy. The POD eigenvector is shown in figure 11. The amplitude of the eigenvector 


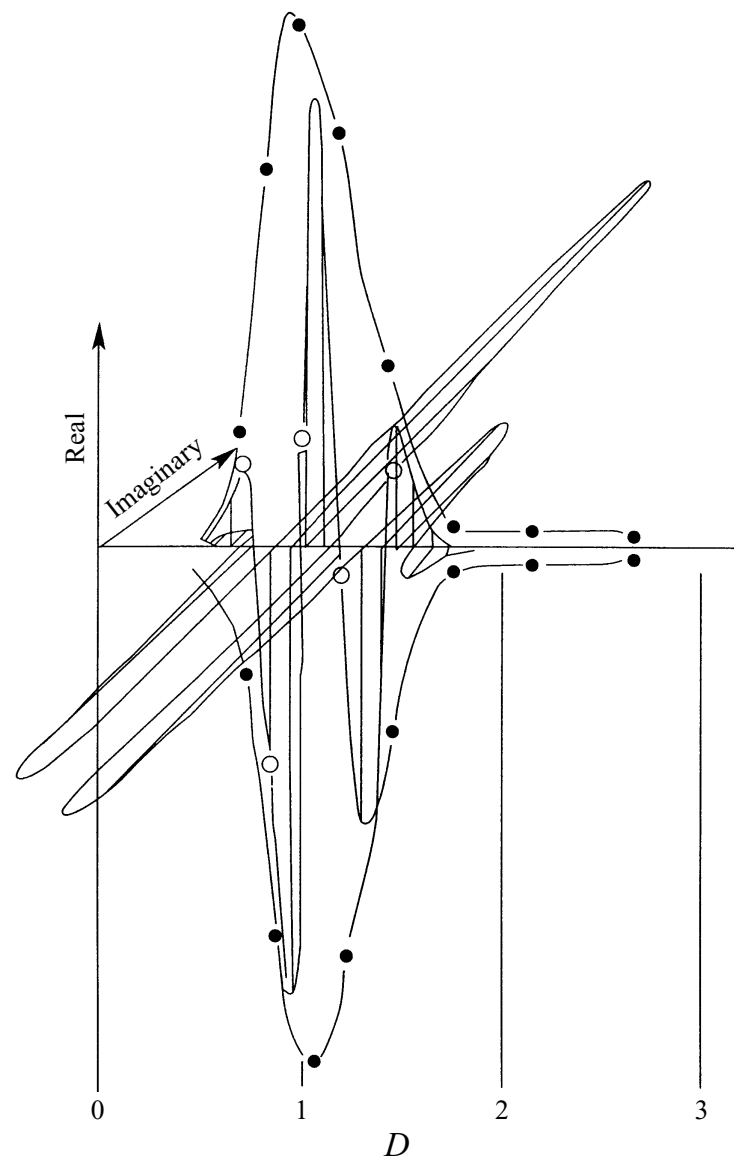

FIGURE 12. Eigenvector $\psi_{1}\left(S t_{D}=1.3, m=0\right)$ corresponding to the largest eigenvalue for the axisymmetric mode at $S t_{D}=1.3$.

is shown at the heavy line through the solid circles. Since each eigenvector is a complex quantity, this amplitude is found by the square root of the sum of the squares of the real and imaginary parts, i.e.

$$
A=\left(\operatorname{Re}(A)^{2}+\operatorname{Im}(A)^{2}\right)^{1 / 2} .
$$

The real part is shown in the vertical plane and the imaginary part is shown in the horizontal plane.

Due to the sparseness of the measurement locations these curves must be generated with some care. In particular, note that neither of the components is just a smooth curve passing through the data. The figure is constructed as follows: First, the amplitude at each point is computed by (5.12) and is plotted as an envelop with a smooth curve drawn through the data. Secondly, realizing that the eigenvector represents a travelling wave train ensures that whenever the imaginary part has a zero crossing the real part must equal the amplitude curve. Whenever the real part has a zero crossing, the imaginary part must equal the amplitude curve. Following these rules a fairly representative curve can be constructed.

It turns out that the form of the eigenvector presented in figure 11 is representative of the axial wave form associated with any particular frequency-azimuthal mode number combination. They all indicate the characteristics of amplification, saturation 


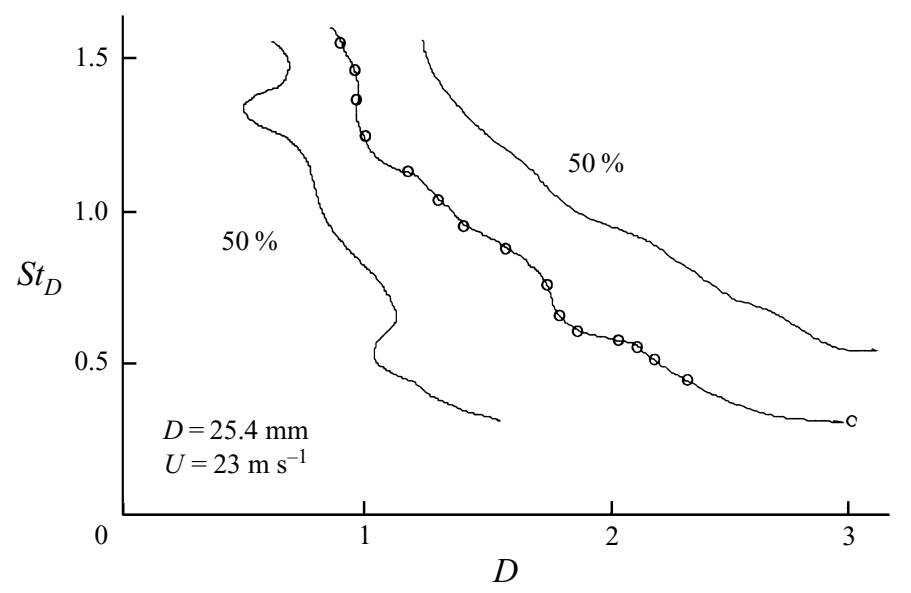

FIGURE 13. Axial location of the peak and $50 \%$ levels for each eigenvector.

and subsequent decay of an instability wave within about three wavelengths. An example of the eigenvector for a different frequency is shown in figure 12. This corresponds to the axisymmetric mode and $S t_{D}=1.3$. This also shows the general character of amplification saturation and decay, but the saturation occurs closer to the jet exit. This is to be expected since the frequency is higher. Note how the magnitude of the eigenvector peaks at $x \sim 0.9 D$, which corresponds to the streamwise location where the near-field pressure spectrum exhibits a peak at this Strouhal number. The peak amplitude has been determined for each frequency component of the $m=0$ mode and plotted on figure 13 along with the $50 \%$ amplitude line. Note that the peak amplitude curve can be approximated by $S t_{D}=D / x$ or $f x / U=1$ which is in good agreement with the shear layer mode of the jet.

Figure 14 shows the $m=1$ component for $S t_{D}=0.5$. It shows the same character as the $m=0$ mode of amplification, saturation and decay. Even the wavy structure is similar. Both real and imaginary parts have their peaks and valleys at approximately the same axial location as in the $m=0$ case. In fact, for a given frequency, the spatial structure of the eigenvectors appears to be similar for all four azimuthal modes studied. This would be expected on the basis of the requirement for orthogonality.

It is tempting to view the results thus far in terms of classical linear modal analysis. Recognizing that the process under investigation is nonlinear, caution must be exercised when interpreting the results. We cannot directly associate an individual eddy with an individual POD mode. This can be resolved by viewing the eigenfunctions as simply building blocks from which an eddy is composed. As the eddy or coherent structure evolves, different eigenmodes will be dominant at different phases in its life cycle (Long 1985; Glauser \& George $1987 a, b$; George 1988; Grinstein et al. 1995). As an example, it is very easy to picture the $m=0$ as an axisymmetric ring vortex and that the $m=1$ component corresponds to a distinct helical or flapping-type motion. In fact, Drubka (1981) found that the jet structure was actually intermittent, jumping back and forth between axisymmetric and right- and left-handed helical modes. In earlier work, Peterson (1978) simultaneously measured the near-field pressure signal with nine separate azimuthally spaced microphones. He found that the signal from each microphone did not line up exactly but that there was some variation in time (Peterson's figure 12). The interpretation given was that the pulses in the pressure signal corresponds to the passage of large-scale coherent structures, consistent with the discussion in $\S 4$. 


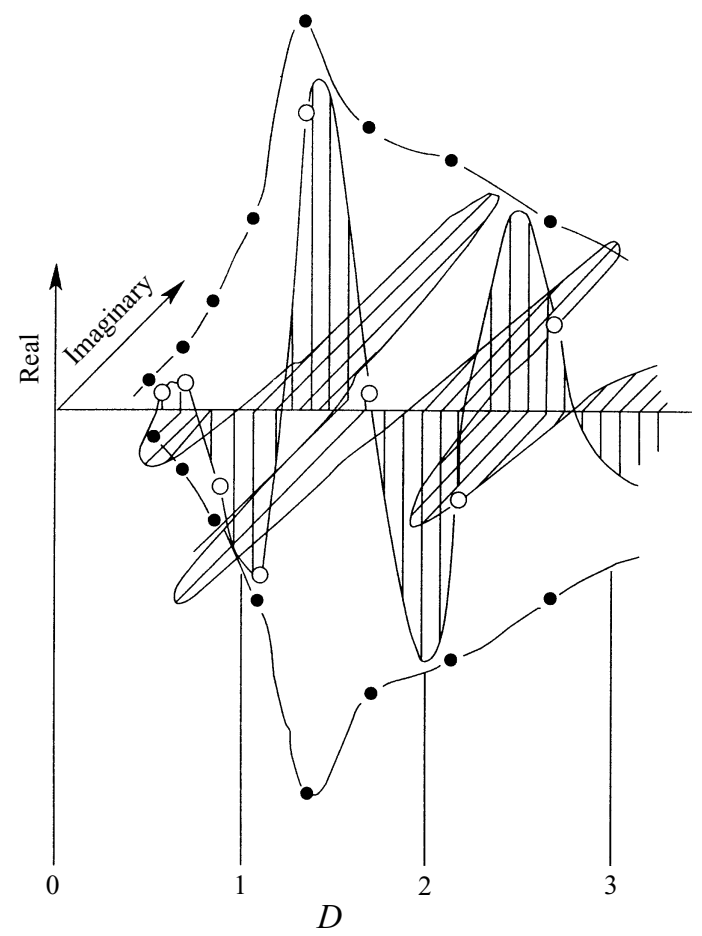

FIGURE 14. Eigenvector $\psi_{1}\left(S t_{D}=0.5, m=1\right)$ corresponding to the largest eigenvalue for the $m=1$ mode at the column mode frequency.

Treating the flow as inhomogeneous in the streamwise direction presents an opportunity to correctly calculate the phase velocity with the POD eigenfunctions. There have been many attempts to measure the phase velocity in turbulent shear flows. Wills (1964) found from hot-wire measurements that the phase velocity was a weak function of frequency; in other words, the wave-like structure was dispersive. Armstrong (1981) measured the phase velocity of the near-field pressure signal and found that the high-frequency components have a constant phase velocity, but at lower frequencies the phase velocity decreased, also indicating dispersive wave-like structures. There are two problems that must be recognized when attempting to measure the phase velocity. As correctly recognized by Wills, the phase velocity must be associated with a wavenumber rather than a frequency. The usual method is to measure the time delay where the maximum correlation between two fixed probes occurs. This gives a convection velocity that is associated with a frequency component which is not what is wanted. The correct method is to fix a time delay and vary the spatial separation between the probes until maximum correlation is achieved. An excellent and more complete discussion of this topic is given by Wills (1964).

The second aspect of the problem, which was not recognized by Wills, is associated with the growth, saturation and decay of the instability waves, i.e. the flow is inhomogeneous in the streamwise direction. Instability waves are used here as an alternative term for the proper orthogonal eigenfunctions. In all previous experiments, including those of Wills, the streamwise direction was treated as if it were homogeneous. That is, a fixed probe is located at one location and a traversable probe is somewhere downstream. Subtle errors can be incurred in this process. If it is imagined that all of the instability waves corresponding to various components are overlain at the various 


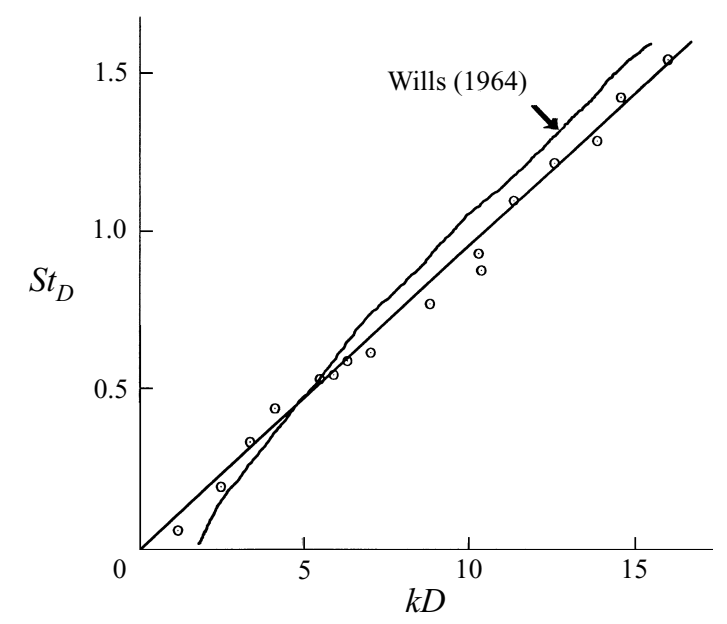

FIGURE 15. Wavenumber-frequency diagram (dispersion relation) of the axial structure of near-field pressure. The phase velocity determined from this is $U_{c} / U_{j}=0.58$ independent of wavenumber shown as the straight solid line. The data of Wills are plotted as well.

probe locations, it is easy to see that the correlation measurements are actually taking place at different axial positions of the growth-saturation-decay of various instability waves. High wavenumbers saturate early in $x$. Therefore, the downstream probe is actually sampling data in the region where the wave is decaying. Similarly the probe system would be positioned in the saturation region for midrange wavenumbers and would be positioned in the growth phase of low wavenumbers. The relative definition of high and low wavenumbers depends on the axial location of the two-probe system. In summary, measurements of waves which are growing or decaying are biased by the rate of growth or decay. Accurate estimates of the phase velocity can be only obtained for instability waves that are close to saturation at the measurement location. This is a possible explanation for the discrepancy between previously reported results.

Phase velocity can easily be determined from the results of this investigation, after all the POD eigenvectors have been constructed. The wavelength of an eigenvector for a given frequency can be measured in the region of peak amplitude (saturation). The phase velocity is simply the quotient of frequency and wavenumber

$$
U_{p}=\omega / k=f \lambda \text {. }
$$

Repeating this process for all measured combinations of frequency and eigenvectors results in the dispersion relationship shown in figure 15, which is a plot of frequency versus wavenumber. The convection velocity for each data point is found from the slope of the vector from the origin to the data point. It is clear that after accounting for experimental error, all the data from the present experiment lie on a straight line passing through the origin. This implies that the wave-like characteristics are nondispersive, i.e. the convection velocity is a constant, independent of frequency. The convection velocity determined from the slope of the line is $U_{c}=13.3 \mathrm{~m} \mathrm{~s}^{-1}$ or $U_{c} / U_{j}=0.58$.

Care must be taken when interpreting these results due to the effects of wavenumber filtering. At a given location there is an entire spectrum of eddy scales, each with its own convection velocity. The convection velocity obtained here is determined from the pressure field that has already been shown to be associated with the large structure due to wavenumber filtering. It should also be kept in mind that inhomogeneities in the radial direction have been averaged out due to the nature of the pressure measurements. 
Since the measurements all took place close to the jet exit $(x<3 D)$ the convection velocity for low wavenumbers (that is, for frequencies less than $f_{c}$ ) are somewhat less accurate because saturation occurs downstream of the last measurement location. Similar errors are inherent in the measurements of Armstrong (1981) and suggest that the measurement is biased because of accuracy limitations.

The wavenumber-dependent convection velocity determined by Wills (1964) is included on figure 15 . His data actually extend to much higher wavenumbers than can be shown on this figure. Clearly the trend of both data sets is different, and we believe this to be due to the reasons discussed previously. However, as noted by Wills, the experimental errors associated with each of these techniques precludes the possibility of giving an accurate value to $U_{c}(k)$. In other words the error bands of each data set overlap.

Peterson (1978) apparently came to the same conclusion regarding the nondispersive nature of jet flow. He showed that a subharmonic disturbance with a wavelength twice the spacing of the paired vortices have a phase speed that matched the convection speed of the vortices before pairing occurred. In other words, there is a definite phase relationship between a particular frequency and its subharmonic. The same result is found in this study. If the eigenvectors for a given frequency and its first subharmonic are plotted together (for instance $S t_{D}=1.0$ and $S t_{D}=0.5$ ), it is found that one wavelength of the $S t_{D}=1.0$ tone exactly fits into half a wavelength of the $S t_{D}=0.5$ tone, such that the zero-crossing points are the same. This pattern is seen for all frequency combinations, i.e. the phase relationship between any frequency and its subharmonic is the same for all pairs having sufficient amplitude to make an accurate comparison. Further discussion of these results in the context of stability theory is given by Liu (1989).

\subsection{Discussion of excited jet results}

One of the reasons for conducting this part of the experiment was to test the POD in a more organized flow field. This was accomplished by exciting the jet acoustically with a mid-frequency horn placed in the anechoic chamber and driven at $3.0 \mathrm{kHz}$ $\left(S t_{D}=3.32\right)$ to produce a level of $90 \mathrm{~dB}$ re $20 \times 10^{-6} \mathrm{~N} \mathrm{~m}^{-2}$ at the nozzle exit. The wavelength of the excitation signal is approximately 4.5 times the nozzle diameter, strongly suggesting that the excitation is axisymmetric (Long 1985). Excitation at this frequency produces a spectrum of the near-field pressure signal which has two discrete tones corresponding to the subharmonics of the excitation signal, namely $1.5 \mathrm{kHz}$ $\left(S t_{D}=1.66\right)$ and $750 \mathrm{~Hz}\left(S t_{D}=0.83\right)$. A simple explanation of this phenomenon can be obtained by considering two successive pairings of ring-like vortices, one associated with the $S t_{D}=1.66$ tone and the other associated with the $S t_{D}=0.83$ tone. For all practical purposes the bulk of the spectral energy can be assumed to be concentrated in these two tones. Hence, it is easier to present the eigenvalues in tabular form rather than in graphical form as was done for the unexcited jet. The results are given in table 1.

Some care must be exercised in interpreting the results in this table. As shown, $73 \%$ of the total energy is concentrated at $S t_{D}=0.83$ and $27 \%$ of the total energy is concentrated at $S t_{D}=1.66$. Consider now the contribution to the total energy from the first POD mode, $\lambda_{1}$. As shown on the table, $\lambda_{1}\left(S t_{D}=0.83,0\right)$ contains $29 \%$ of the total energy. Summing up the first four azimuthal modes of $\lambda_{1}$ results in $71 \%$ of the total energy, nearly $100 \%$ of the energy concentrated at this frequency. By comparison, summing up the first four azimuthal modes of $\lambda_{1}$ at $S t_{D}=1.66$ results in $15 \%$ of the total which is still more than $50 \%$ of the energy at this frequency. The data presented in the table clearly indicate that all the energy is not located in the $m=0$ mode as would be expected from a model of axisymmetric vortices. It was expected that the 

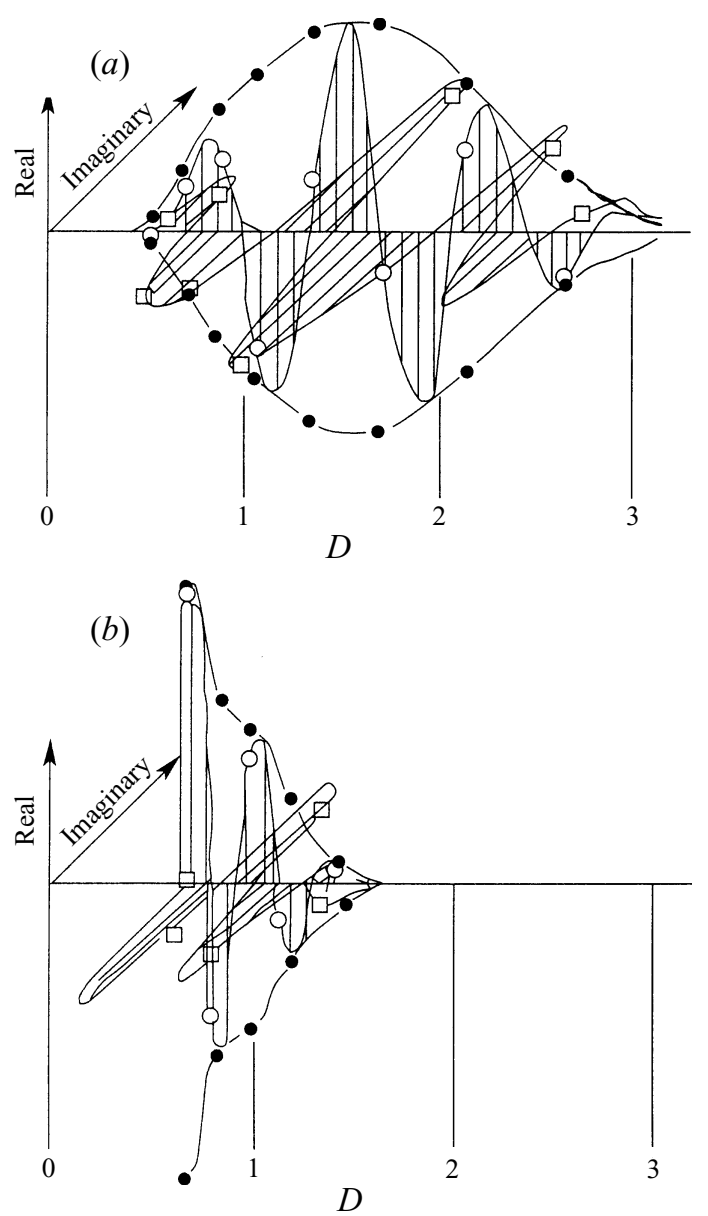

FIGURE 16. (a) Eigenvector $\psi_{1}\left(S t_{D}=0.83, m=0\right)$ corresponding to the largest eigenvalue for the axisymmetric mode at $f_{e} / 4$ in the excited jet. $(b)$ Eigenvector $\psi_{1}\left(S t_{D}=1.66, m=0\right)$ corresponding to the largest eigenvalue for the axisymmetric mode at $f_{e} / 2$ in the excited jet.

\begin{tabular}{ccc}
\hline & $S t_{D}=0.83$ & $S t_{D}=1.66$ \\
Fraction of total energy & 0.73 & 0.27 \\
Mode: $\quad m=0$ & 0.29 & 0.06 \\
$m=1$ & 0.25 & 0.03 \\
$m=2$ & 0.14 & 0.02 \\
$m=3$ & 0.04 & 0.04 \\
Sum of first four azimuthal & 0.72 & 0.15 \\
modes of $\left|\lambda_{1}(\omega, m)\right|^{2}$ & \\
TABLE 1. Comparison of azimuthal decomposition of $\left|\lambda_{1}(\omega, m)\right|^{2}$ and the total mean square \\
of the spatially integrated signal \\
\hline
\end{tabular}

excitation would result in a more organized pattern with less jitter than was assumed to be associated with the $m=1$ and higher azimuthal mode components. Apparently what has happened is that the excitation has phase locked the signal but not in the axisymmetric mode. The ring-like structures are still apparent in the signal but have become stretched and distorted in the axial direction (Peterson 1978). The real and 
imaginary parts of the first eigenvector for the $S t_{D}=0.83$ tone are shown in figure 16(a). It shows the same general character as in the unexcited jet. The phase velocity is computed as $U_{c}=0.59 U_{j}$. The first eigenvector for the $S t_{D}=1.66$ tone is shown in figure 16(b). Only the decay region is seen; apparently the wave saturated closer to the exit than the first measurement location. The phase velocity for this wave is also computed as $U_{c}=0.59 U_{j}$. As in the unexcited case, the first eigenvector is dominant at each of the two frequencies. Since the total energy is concentrated at these two frequencies, the entire jet structure can be described by these two dominant eigenvectors.

\section{The shot effect}

By combining the shot-effect decomposition originated by Rice (1944) with the POD Lumley (1981) was able to develop a useful method that allows a quantitative measure of coherent structures in turbulent flows. The shot effect was considered by Rice (1944) for studying the statistics of vacuum tube noise where a pulse is emitted every time an electron reached the anode. Since that time, several researchers have used Rice's theory to reconstruct eddies from POD data (Long \& Arndt 1985; Herzog 1986; Glauser et al. 1985, 1987; Moin \& Moser 1989). In his theory, the measured spectrum is the Fourier transform of an individual pulse and a characteristic event can be reconstructed from the inverse Fourier transform of the measured spectrum of the shot noise. Lumley extended this idea for continuously variable amplitude and defined the inverse Fourier transform of the spectrum associated with the largest eigenvalue as a 'characteristic eddy'. In the present work, this function is defined as a characteristic event.

In most experiments where correlation techniques are used, a fixed probe is located at a specific point and another probe is traversed. This method can produce correlation lengths and amplitudes for individual frequency components, but it cannot produce the phase relationship between frequencies; all frequencies are phase referenced to the location of the fixed probe. In the present experiment the phase is chosen so that the downstream convection is preserved. This information can be used as an input to the shot-effect decomposition to reconstruct a typical signal or characteristic event.

Only the dominant POD eigenfunction will be used so that a POD mode estimate of the pressure signal is written as a function of time and space $x$ (the $\theta$-dependence is suppressed for now) as

$$
p^{1}=p^{1}(x, t)
$$

where the superscript 1 denotes the first POD mode. It is supposed that this signal can be decomposed in time as

$$
p^{1}(x, t)=f(x, t) * g(t),
$$

where the $*$ represents a convolution, $f$ is the characteristic event, and $g$ is the random strength function. Since the signal is a stationary function, $g$ must also be a stationary function which should satisfy ergodicity: uncorrelated in non-overlapping intervals. Choosing a strength function to have the same correlation as white noise allows

$$
\overline{g(t) g(t-\tau)}=\overline{a^{2}} \delta(\tau),
$$

where $\overline{a^{2}}$ is the average energy associated with each individual event.

An estimate of the cross-spectrum utilizing the first POD mode can be written as

$$
\Phi_{(1)}\left(x, x^{\prime} ; \omega\right)=\lambda_{(1)}^{2}(\omega) \psi_{(1)}(x, \omega) \psi_{(1)}\left(x^{\prime} ; \omega\right)^{H},
$$

where the superscript $H$ stands for Hermitian transpose. 
Using (6.3) it can also be shown that

$$
\Phi_{(1)}\left(x, x^{\prime} ; \omega\right)=\overline{a^{2}} F\{f(x, t)\} F^{*}\left\{f\left(x^{\prime}, t\right)\right\},
$$

where $F\{\cdot\}$ stands for the Fourier transform of the quantity inside the curly brackets. For the particular case of $x=x^{\prime}$, a direct association between (6.4) and (6.5) can be made so that

$$
\left(\overline{a^{2}}\right)^{1 / 2} F\{f(x, t)\}=\lambda_{1}(\omega) \psi_{1}(x ; \omega) \mathrm{e}^{\mathrm{i} \Theta(\omega)}
$$

from which $f$ can be found by an inverse Fourier transform.

It shall be shown that using (6.6) directly with $\Theta(\omega)=0$ leads to a result entirely consistent with the downstream convection of turbulent structures. This appears to be consistent with the results of Herzog (1986) and Moin \& Moser (1989). Herzog found that the case of zero phase shift produced the most compact form of the typical eddy. Herzog also showed that the principle features of the typical eddy did not change when phase shifts other than 0 were selected. Moin \& Moser (1989) found that their $u$ velocity compactness condition was satisfied by the zero-phase eddy. Moin \& Moser (1989) also showed, for their two-dimensional case, that the compactness conditions produced the same results as when the bi-spectrum was utilized. Hence, in this study, only the $\Theta(\omega)=0$ case will be examined.

Equation (6.6), with $\Theta(\omega)=0$, is given by

$$
\left(\overline{a^{2}}\right)^{1 / 2} F\{f(x, t)\} \simeq \lambda_{(1)}(\omega) \psi_{(1)}(x, \omega),
$$

where the eigenvector $\psi_{(1)}$ is determined from (5.10). For each azimuthal mode, (6.7) is to be evaluated eight times, once for each of the eight measurement locations. Remember that the characteristic event is a function of both downstream distance and time

$$
f=f(x, t ; m)
$$

for each azimuthal mode $m$. It is evident that a shot-effect decomposition and subsequent inverse transform could be performed for the azimuthal mode as well. This would lead to a characteristic event of the form

$$
f=f(x, t, \theta) .
$$

This is not done here since it is felt that the structure is more clearly evident when displayed in the form described by (6.8) especially since three independent variables and one dependent variable cannot be plotted conveniently. It is easy, however, to plot (6.8) as a contour map because it consists of only two independent variables.

As previously discussed, the spectrum for the excited jet experiment contains two subharmonics of the excitation frequency, $S t_{D}=3.32$. The first subharmonic is due to an instability wave that saturates close to the nozzle lip. The second subharmonic is due to the instability wave that saturates further downstream. In this case the characteristic event for the flow would be associated with the pairing event that results in the formation of the second subharmonic.

The result for the excited case is shown in figure 17 as a contour map in the form described by (6.8). Only the axisymmetric mode is considered. Time is plotted horizontally and normalized by $U_{j}$ and $D$. Axial distance is plotted obliquely for $0<x / D<3$, and amplitude is plotted vertically. As expected, the high-frequency tone occurs near the jet exit and the low-frequency tone occurs further downstream. This is usually associated with pairing where one eddy slows down and the one behind it speeds up, which leads to the coalescence. This apparently does not happen here. The even-numbered humps seem to persist and convect downstream at a constant speed 


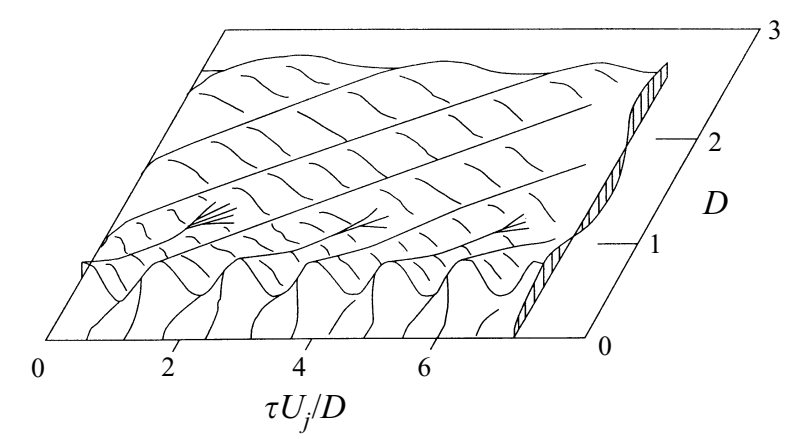

FIGURE 17. Characteristic event in the excited jet, axisymmetric mode.

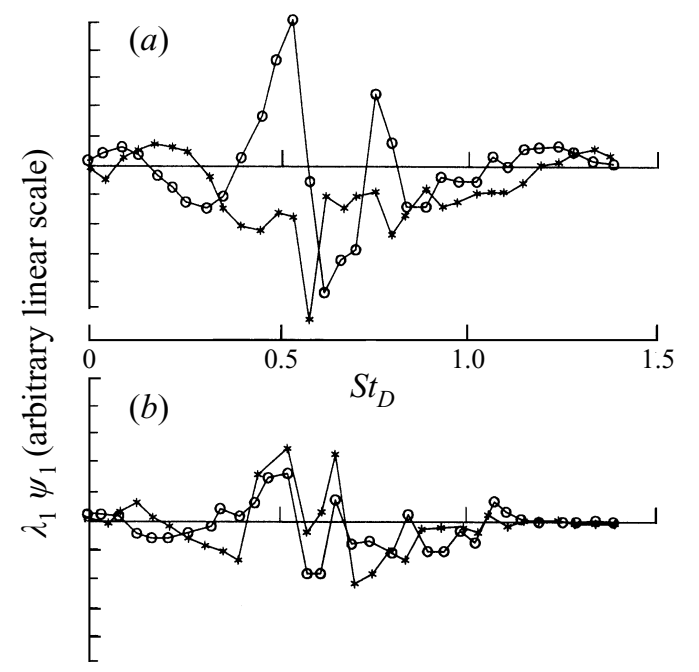

FIGURE 18. Characteristic spectra to be used in (6.7). $x=1.37 D$. Open circles are the real part; asterisks are the imaginary part. (a) $m=0,(b) m=1$.

while odd-numbered humps simply die away beyond one diameter. As previously discussed, an individual ridge in $\{t, x / D\}$-space of the pressure signal cannot be directly related to an individual eddy. However, Long (1985) conjectured that the data in figure 17 are consistent with a model of two non-adjacent eddies stretching and tearing a third eddy that was originally between them as proposed by Moore \& Saffman (1975). This third vortex gets stretched into a braid and the vorticity is entrained into the first two eddies. Bernal (1981) found evidence for this by a flow visualization experiment. It was a rather rare occurrence compared to the vortex pairing, but it is possible that the excitation in the present experiment locked onto this phenomenon and resulted in the pattern seen in figure 17.

After consideration of this relatively simple experiment it is now easier to interpret the results for the unexcited jet. Before discussing the contour plots described by (6.9) some of the intermediate results are considered. Figure 18 is an example of the frequency function for use on the right-hand side of (6.7). Both real and imaginary parts are shown. The inverse Fourier transform of this function yields the characteristic event at the specified location. Note again that this is for $\Theta(\omega)=0$. The result is shown in figure 19. It is real valued and can be thought of as a typical time trace. When this operation is carried out at each of the seven other locations, the result can be displayed 

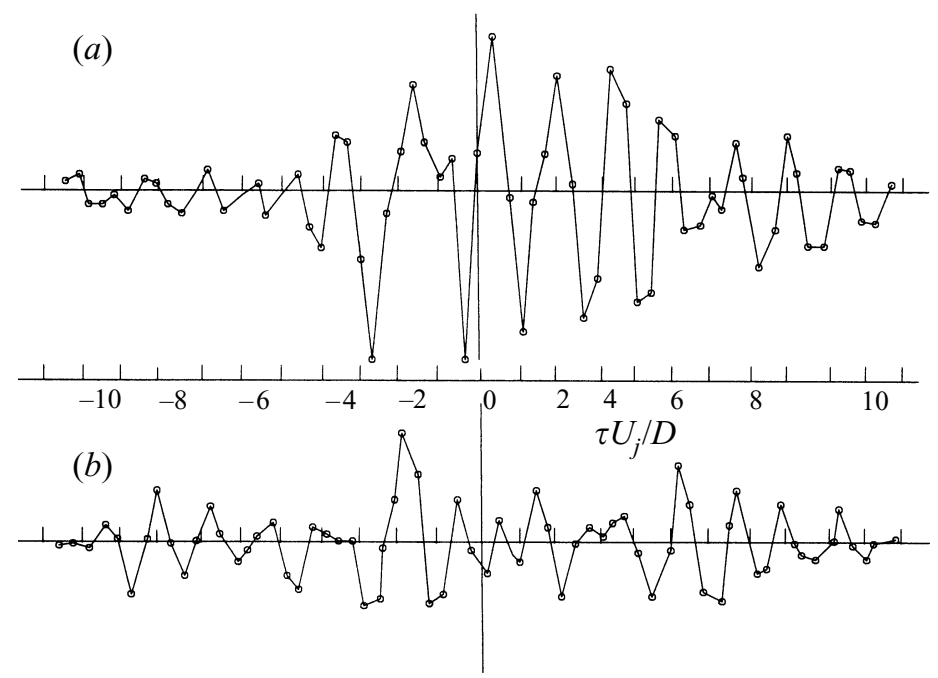

FIgURE 19. Characteristic signals obtained from (6.7). $x=1.37 D ;(a) m=0,(b) m=1$.
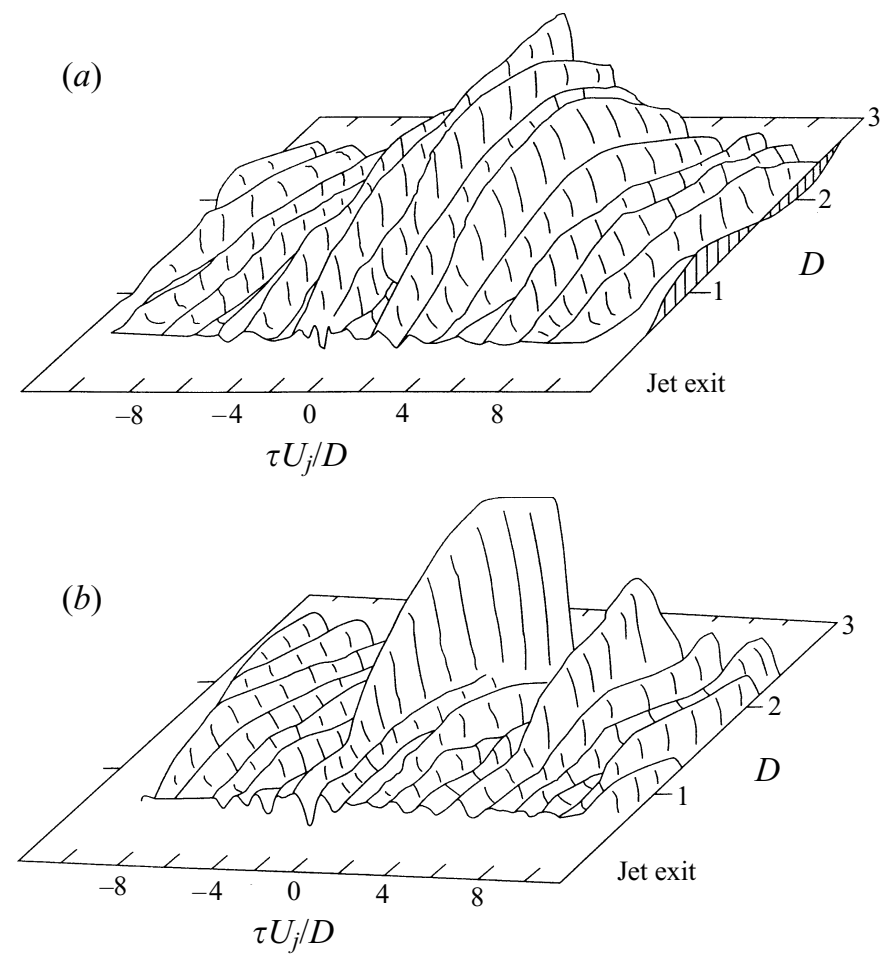

FIGURE 20. Characteristic event for (a) the $m=0$ and $(b)$ the $m=1$ mode viewed obliquely.

as a contour map similar to figure 17. Figure $20(a)$ is the result for the axisymmetric mode and figure $20(b)$ is the result for the $m=1$ mode.

Evidently the characteristic event described by these figures consists of a sequence of pressure ridges of varying amplitude. One can see evidence for the coalescence and annihilation of certain ridges as the structure progresses downstream. If each of these ridges is interpreted as the passage of a vortex structure this phenomenon can be 
(a)

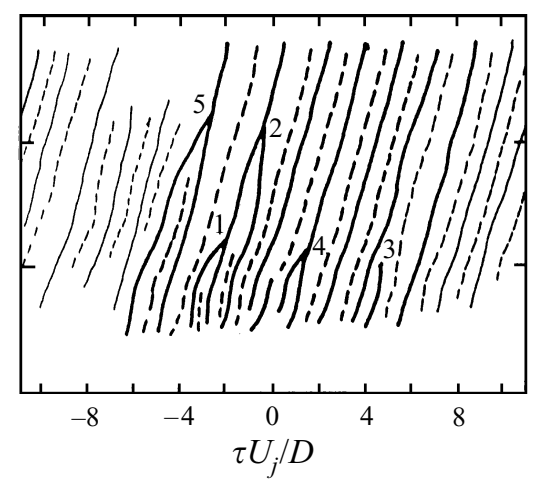

(b)

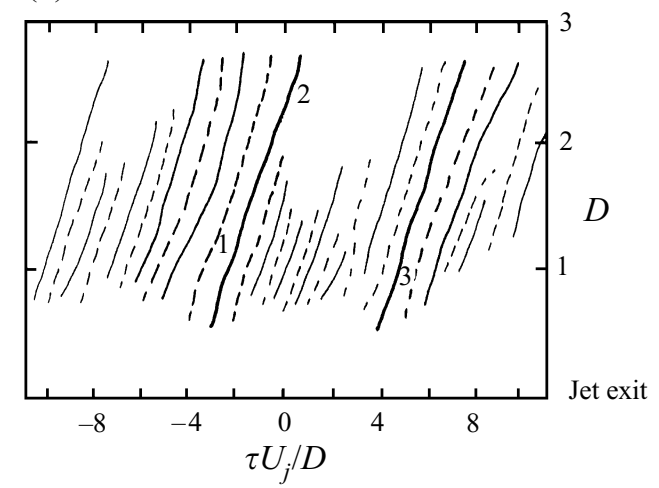

Figure 21. (a) Plan view of (a) figure 20(a), $m=0$, (b) figure 20(b) $m=1$.

thought of as a vortex pairing or vortex tearing. For the most part however, each ridge that forms convects downstream and does not interact with its neighbours.

Another way to look at these data is to view the contour plots directly from above. This yields the typical form of an $(x, t)$-diagram. The axisymmetric mode corresponding to figure $20(a)$ is shown on figure $21(a)$. The $m=1$ mode corresponding to figure $20(b)$ is shown on figure $21(b)$. The solid lines indicate the location of the peaks and the dotted lines the valleys of the signal. Heavier lines indicate high peaks and light lines indicate low peaks. A void in the plot indicates that no peak or valley is discernable because the level is too low. The figures clearly demonstrate that the peaks and valleys are associated with a convected structure. The convection velocity determined here is the same as the phase velocity determined from the eigenfunctions directly as $U_{c}=0.58 U_{j}$. This shows that the reconstruction using zero phase is a physically realistic structure, consistent with the results of Herzog (1986) and Moin \& Moser (1989).

From an analysis of the contour plots and the associated diagrams for both the $m=0$ and $m=1$ modes, the following statements concerning the large-scale turbulence can be made:

(i) The initial growth of the large pulse in the $m=1$ mode occurs just downstream of a pairing event in the $m=0$ mode. This location is denoted by a 1 in all the relevant figures. Further downstream along the same ridge another pairing event in the $m=0$ mode occurs and the large ridge in the $m=1$ mode is increased. This location is denoted by a 2. This suggests that this 'triple vortex interaction' is responsible for the general breakdown of the axisymmetric mode into higher-order modes. It appears that the initially ring-like vortices breakdown because of incomplete pairing.

(ii) Figure 21 (a) suggests that there are also 'double vortex interactions' occurring. This happens at three distinct locations. These are denoted by 3, 4, 5. Apparently a double pairing is three times more common than a triple pairing.

(iii) The point of this discussion is to show that although this is a low-Reynoldsnumber 'clean' jet the large-scale structure is not composed of a sequence of vortex pairings that occur at successive downstream locations. The structure is more complicated, being made up of both pairings and triplings occurring erratically in time and incomplete in azimuthal angle.

Having interpreted the characteristic event $f(x)$ as being associated with vortex pairings it is now of interest to discuss how many of these events occur in a given period. Thus far, knowledge of this structure is limited to the average energy. There are many ways in which this characteristic event could be divided that is consistent with 
the measured energy. The event could be extremely violent but relatively rare or it could be less violent and much more frequent. The obvious goal is to determine which of these is correct.

In order to do this an extension of Campbell's theorem (Rice 1944) must be discussed. The extension gives a relation for the $n$th moment of the probability density function. Specifically it can be shown that, for $\bar{p}=0$,

and that

$$
\begin{aligned}
& \overline{p^{2}}=\nu \overline{a^{2}} \int_{-\infty}^{\infty} f^{2}(\tau) \mathrm{d} \tau \\
& \overline{p^{4}}=\nu \overline{a^{4}} \int_{-\infty}^{\infty} f^{4}(\tau) \mathrm{d} \tau,
\end{aligned}
$$

where $v$ in both cases represents the number of occurrences of the event $f(t)$ per unit time. The rate of occurrence is found by squaring (6.10) and dividing by (6.11) to get

$$
\nu=\left\{\frac{\overline{a^{4}}}{\overline{a^{2}}}\right\}\left\{\frac{\overline{p^{2}}}{\overline{p^{4}}}\right\} \int_{-\infty}^{\infty} f^{4}(\tau) \mathrm{d} \tau /\left\{\int_{-\infty}^{\infty} f^{2}(\tau) \mathrm{d} \tau\right\}^{2} .
$$

To actually calculate $v$ from (6.12), the moments of $a$ and $p$ must be investigated.

Since $\bar{p}=0$ the second factor in (6.12) is actually the inverse of the kurtosis. An application of the central-limit theorem indicates that the probability density of $p$ will approach a normal distribution and hence the kurtosis will approach 3 as $v$ approaches infinity (Tennekes \& Lumley 1972).

The same statement cannot be made regarding the distribution of $a$ because the mean value of $a$ is not zero. The value of the fourth moment divided by the square of the second moment depends strongly on $a$ even though the higher moments of the probability density function are distributed normally. To proceed, two cases must be considered. These are given by $\bar{a} / \sigma \rightarrow \infty$, and $\bar{a} / \sigma \rightarrow 0$ where $\sigma$ is the standard deviation of the event amplitude.

The first case corresponds to a physical situation where the size and shape of all events are the same. The value of the first factor in (6.12) will approach 1 . This is the desirable situation because it is hoped that all events will be roughly the same size. The second case implies that the amplitude of the events is a random variable centred about a zero mean. This situation is less desirable because this randomness does not relate well to the physical notion of a characteristic event. If its distribution is assumed Gaussian with zero mean the first factor in (6.12) is the kurtosis and will have a value of 3. Considering both of these cases places bounds on the estimate of $\nu$.

In accordance with the assumption that all events are similarly sized the choice of $a=$ constant is acceptable and (6.12) can be simplified to

$$
\left.\left.\nu=\frac{1}{3} \int_{-\infty}^{\infty} f^{4}(\tau) \mathrm{d} \tau /\left\{\int_{-\infty}^{\infty} f^{2}\right) \tau\right) \mathrm{d} \tau\right\}^{2} .
$$

where the pressure is assumed Gaussian.

Both numerator and denominator of (6.13) have been evaluated at each of the eight measurement locations for both the $m=0$ and $m=1$ modes. The rate of occurrence that is calculated shows some variance from location to location, but this is unimportant and probably due to the inability to calculate the fourth moment accurately. The average value determined from these 16 individual values is found to be

The inverse of this relation is

$$
\begin{gathered}
\nu D / U_{j}=0.075 \\
\tau U_{j} / D=13.3
\end{gathered}
$$


and represents the average time between occurrences. We estimate this value to be accurate to within $20 \%$. This implies that the events described by figures $20(a)$ and $21(b)$ repeat at this average rate. Since a triple pairing only occurs once within a characteristic event, it will occur once every 13.3 dimensionless time units. Since double interactions occur three times more frequently, they will occur once every 4-5 dimensionless time units.

The results that have been described in this section are believed to be typical of all low-Reynolds-number low-Mach-number jets. There will of course be differences depending on individual geometry and initial conditions as has been demonstrated by Ho \& Huerre (1984).

The results here show that the shot-noise decomposition seems to be applicable to periodically excited flows. In general, the shot-noise method has several disadvantages as discussed by Berkooz et al. (1993) and Sirovich (1989).

\section{Conclusions}

The objective of this work was to examine the inhomogeneity in the streamwise direction for a round jet through the application of POD. For the first time POD was applied to the pressure field surrounding the jet. This has certain advantages, such as ease of measurement and greatly simplified data analysis as compared to applying POD to the velocity field. For example, we have been able to study the streamwisetemporal structure of the jet with a minimum number of spatial grid points. At any particular frequency, the POD eigenfunction grows, saturates, and decays all within about three wavelengths as it convects downstream. High frequencies saturate early in $x$ and low frequencies saturate further downstream. Due to the complexity of applying the POD, similar data have not been obtained from the velocity field.

Since the eigenfunctions are broken down into discrete frequency components, the phase velocity can be determined using the measured wavelength of the POD eigenfunctions near the saturation point. This is an important result since all previous measurements of phase velocity suffer from the probe being located on different phases of the growth-saturation-decay cycle. There is a bias when the measurement takes place in the growth or decay phase; only in the saturation region is a true estimate of phase velocity obtained. The phase velocity determined from the proper orthogonal decomposition was found to be $U_{p}=0.58 U_{j}$, independent of frequency. This is the first time that phase velocity has been determined giving due consideration to the streamwise inhomogeneity in the initial region of the jet.

POD also extracts the energy of pressure fluctuations associated with each azimuthal mode. The axisymmetric or $m=0$ mode contains $50 \%$ of the total; the $m=1$ mode $23 \%$; the $m=2$ mode $14 \%$; and the $m=3$ mode $5 \%$. We consider this to be the baseline situation because the jet was operated at low Reynolds number and low Mach number. If either of these parameters increases, it is expected that the higher-order modes will contain a greater proportion of the total, consistent with the presence of smaller scales.

The characteristic signal form (characteristic eddy or characteristic event) was reconstructed from the results of POD using the shot-effect decomposition. Results showed that even at this low Reynolds number, vortex pairing does not occur in a repeatable fashion at a specific downstream location but is random in both time and space. Vortex tripling occurs one third as often and is associated with a strong $m=1$ component suggesting that the jet is beginning to break down into smaller scales.

The results presented here demonstrate the utility of applying POD to the pressure field. This sets the stage for practical opportunities for POD-based control strategies. 
This research was supported by the Air Force Office of Scientific Research and the Office of Naval Research. The authors are grateful to Colonel Lowell Ormand, AFOSR, Dr Michael Reischman, ONR and Dr Edwin Rood, ONR who were contract monitors during different phases of this work. M. N. Glauser would like to particularly thank Dr J. M. Seiner at the NASA Langley Research Center for his support. R. E. A. Arndt would also like to acknowledge the assistance of the Alexander von Humboldt Foundation in the preparation of this paper. All of the authors would like to express their gratitude to the reviewers for their many helpful comments and suggestions.

\section{REFERENCES}

Armstrong, R. R. 1981 Influence of Mach number on coherent structure relevant to jet noise. AIAA J. 19, 677.

Arndt, R. E. A. \& George, W. K. 1974 Investigation of the large scale coherent structure in a jet and its relevance to jet noise. Proc. Second Interagency Symp on University Research in Transportation Noise, North Carolina State University, Raleigh, NC, June 5-7.

Aubry, N., Holmes, P., Lumley, J. L. \& Stone, E. 1988 The dynamics of coherent structures in the wall region of turbulent boundary layer. J. Fluid Mech. 192, 115.

Berkooz, G., Holmes, P. \& Lumley, J. L. 1993 The proper orthogonal decomposition in the analysis of turbulent flows. Ann. Rev. Fluid Mech. 25, 539-75.

Bernal, L. P. 1981 The coherent structure of turbulent mixing layers. CIT Report, June, 1981.

Carlson, H. A., Berkooz, G. \& Lumley, J. L. 1995 Direct numerical simulation of flow in a channel with complex time dependent wall geometries; a pseudo spectral method. J. Comput. Phys. 121, 155.

Chambers, D. H., Adrian, R. J., Moin, P., Stewart, D. S. \& Sung, H. J. 1988 Karhunen-Loeve expansion of Burgers model of turbulence. Phys. Fluids A 31, 2573.

Crighton, D. G. 1981 Acoustics as a branch of fluid mechanics. J. Fluid Mech. 106, 261.

Crow, S. C. \& Champagne, F. H. 1971 Orderly structure in jet turbulence. J. Fluid Mech. 48, 547.

Delville, J. 1995 La decomposition orthogonale aux valeurs propres et l'analyse de l'organisation tirdimensionelle des ecoulements turbulents cisailes libres. Thesis, University of Poitiers, France.

Deville, J., Bellin, S. \& Bonnet, J. P. 1991 Use of the proper orthogonal decomposition in a plane turbulent mixing layer. In Turbulence and Coherent Structures (ed. O. Metais \& M. Lesieur), pp. 75-90. Kluwer.

DrubKa, R. E. 1981 Instabilities in near field of turbulent jets and their dependence on initial conditions and Reynolds number. $\mathrm{PhD}$ thesis, Illinois Institute of Technology.

Fisher, M. J., Harper-Bourne, M. \& GlegG, S. A. L. 1977 Jet engine noise source location: the polar correlation technique. J. Sound Vib. 51, 23.

Fuchs, H. V. 1972 Space correlations of the fluctuating pressure in subsonic turbulent jets. J. Sound Vib. 23, 77.

GEORGE, W. K. 1988 Insight into the dynamics of coherent structures from a proper orthogonal decomposition. Symp. on Near Wall Turbulence, Dubrovnik, Yugoslavia, May 16-20.

George, W. K., Beuther, P. D. \& Arndt, R. E. A. 1984 Pressure spectra in turbulent free shear flows. J. Fluid Mech. 148, 155.

GlausER, M. N. 1987 Coherent structures in the axisymmetric jet shear layer. PhD dissertation, SUNY at Buffalo.

Glauser, M. N. \& George, W. K. $1987 a$ Orthogonal decomposition of the axisymmetric jet mixing layer including azimuthal dependence. In Advances in Turbulence (ed G. Comte-Bellot \& J. Mathieu), pp. 357-366. Springer.

Glauser, M. N. \& George, W. K. $1987 b$ An orthogonal decomposition of the axisymmetric jet mixing layer utilizing cross wire measurements. Proc. Sixth Symp. Turbulent Shear Flows, Toulouse, France, p. 10.1.1.

Glauser, M. N. \& George, W. K. 1992 Application of multipoint measurements for flow characterization. Expl Thermal Fluid Sci. 5, 617. 
Glauser, M. N., Leib, S. J. \& George, W. K. 1985 Coherent structures in the axisymmetric turbulent jet mixing layer. Fifth Symp. on Turbulent Shear Flows, Ithaca, NY, August 7-9.

Glauser, M. N., Leib, S. J. \& George, W. K. 1987 Coherent structures in the axisymmetric jet mixing layer. In Turbulent Shear Flows 5 (ed. F. Durst et al.), p. 134. Springer.

Glezer, A., Kadioglu, Z. \& Pearlstein, A. J. 1989 Development of an extended proper orthogonal decomposition and its application to a time periodically forced plane mixing layer. Phys. Fluids A 1, 1363.

Grinstein, F. F., Glauser, M. N. \& George, W. K. 1995 Vorticity in jets. In Fluid Vortices (ed. S. I. Green), p. 65. Kluwer.

Herzog, S. 1986 The large scale structure in the near wall region of turbulent pipe flow. $\mathrm{PhD}$ dissertation, Cornell University.

Ho, C. M. \& Huerre, P. 1984 Perturbed free shear layers. Ann. Rev. Fluid Mech. 16, 365-424.

Hussain, A. K. M. F. 1983 Coherent structures reality and myth. Phys. Fluids 26, 2816.

Hussain, A. K. M. F. 1986 Coherent structures and turbulence. J. Fluid Mech. 173, 303.

Hussain, A. K. M. F. \& Zaman, K. B. M. Q. 1978 The free shear layer tone phenomenon and probe interference. J. Fluid Mech. 87, 349.

Jones, B. B., Adrian, R. J., Nithianandan, C. K. \& Planchon, H. P. 1979 Spectra of turbulent static pressure fluctuations in jet mixing layers. AIAA J. 17, 449.

Kim, H. J. 1983 Sound source distributions in excited and unexcited turbulent jets. MS. thesis, University of Minnesota.

Ko, N. W. M. \& Davies, P. O. A. L. 1971 The near field within the potential cone of subsonic cold jets. J. Fluid Mech. 50, 49.

LighthiLL, M. J. 1954 On sound generated aerodynamically. II. Turbulence as a source of sound. Proc. R. Soc. Lond. A 222, 1.

LiU, J. T. C. 1989 Coherent structures in transitional and turbulent free shear flows. Ann. Rev. Fluid Mech. 21, 285.

Loeve, M. 1978 Probability Theory, 4th edn. Springer.

LoNG, D. F. 1985 Noise radiation and coherent structure in turbulent jets: viscous and compressibility effects. PhD thesis, University of Minnesota.

Long, D. F. \& Arndt, R. E. A. 1984 Jet noise at low Reynolds number. AIAA J. 22, 187.

Long, D. F. \& ARNDT, R. E. A. 1985 Orthogonal decomposition of pressure fluctuations surrounding a turbulent jet. Proc. Turbulent Shear Flow Conf., Ithaca, NY, August.

Long, D. F., Kim, H. J. \& ARndt, R. E. A. 1985 Controlled suppression or amplification of turbulent jet noise. AIAA J. 23, 828 .

LumLey, J. L. 1967 The structure of inhomogeneous turbulent flows. In Atmospheric Turbulence and Radio Wave Propagation (ed. A. M. Yaglom \& V. I. Tartarsky), p. 166. Nauka, Moscow.

Lumley, J. L. 1970 Stochastic Tools in Turbulence. Academic.

Lumley, J. L. 1981 Coherent Structures in Turbulence (ed. R. E. Meyer), p. 215. Academic.

Manceau, R. 1995 Etude de systèmes dynamiques basès sur la décomposition orthogonale aux valeurs propres en couche de mélange turbulent. Diplome D'Etudes Approfondies, CEAT/LEA Poitiers France.

MichalKe, A. 1977 On the effect of spatial source coherence on the radiation of jet noise. J. Sound Vib. 55, 377.

Morn, P. 1984 Probing turbulence via large eddy simulation. AIAA Paper 84-0174.

Moin, P. \& Moser, R. D. 1989 Characteristic eddy decomposition of turbulence in a channel. J. Fluid Mech. 200, 471.

Moore, C. J. 1977 The role of shear layer instability waves in jet exhaust noise. J. Fluid Mech. 80, 321.

Moore, D. W. \& Saffman, P. G. 1975 The density of organized vortices in turbulence mixing layers. J. Fluid Mech. 69, 465.

Morse, P. M. \& Ingard, K. U. 1968 Theoretical Acoustics. McGraw-Hill.

Peterson, R. A. 1978 Influence of wave dispersion on vortex pairing in a jet. J. Fluid Mech. 89, 469.

Rajaee, M., Karlson, S. \& Sirovich, L. 1994 Low-dimensional description of free-shear-flow coherent structures and their dynamical behaviour. J. Fluid Mech. 258, 1. 
RAN, B. \& KATZ, J. 1994 Pressure fluctuations and their effect on cavitation inception within water jets. J. Fluid Mech. 262, 223.

RicE, S. O. 1944 Mathematical analysis of random noise. Bell System Tech. J. 23, 24.

SeINER, J. M. \& GILINSKY, X. 1995 Nozzle thrust optimization while reducing jet noise. CEAS/AIAA $-95-149$.

Sirovich, L. 1989 Chaotic dynamics of coherent structures. Physica D 37, 126.

Sirovich, L. \& Rodriguez, J. D. 1987 Coherent structures and chaos. Phys. Lett. A 120, 211.

Tam, C. K. W. 1995 Supersonic jet noise. Ann. Rev. Fluid Mech. 27, 17.

Tennekes, H. \& Lumley, J. L. 1972 A First Course in Turbulence. MIT Press.

Ukeiley, L. \& Glauser, M. N. 1995 Dynamics of large scale structures in a plane turbulent mixing layer. Clarkson University Rep. MAE-311.

Ukeiley, L., Varghese, M., Glauser, M. \& Valentine, D. 1992 Multifractal analysis of a lobed mixer flowfield utilizing the proper orthogonal decomposition. AIAA J. 30, 1260.

WiLls, J. A. B. 1964 On convection velocity in turbulent shear flows. J. Fluid Mech. 20, 417.

Zheng, X. \& Glauser, M. N. 1991 A low dimensional dynamical systems description of coherent structures in the axisymmetric jet mixing layer. Clarkson University Rep. MAE-247. 